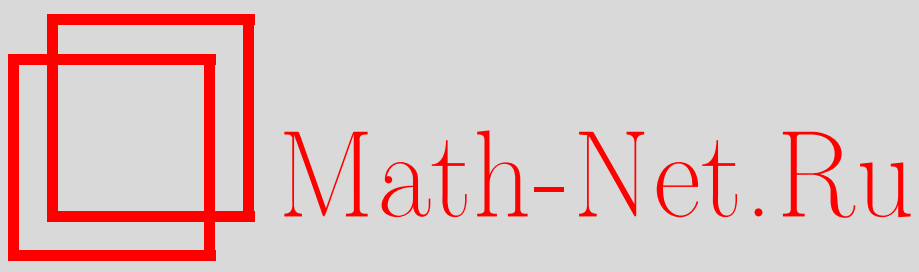

В. В. Щиголев, Конечная базируемость $T$-пространств над полями нулевой характеристики, Изв. РАН. Сер. матем., 2001, том 65, выпуск 5, 191-224

DOI: https://doi.org/10.4213/im362

Использование Общероссийского математического портала Math-Net.Ru подразумевает, что вы прочитали и согласны с пользовательским соглашением

http://www . mathnet.ru/rus/agreement

Параметры загрузки:

IP: 107.22 .136 .117

26 апреля 2023 г., 18:34:30 
УДК 519.48

\author{
В.В. Щиголев
}

\title{
Конечная базируемость $T$-пространств над полями нулевой характеристики
}

\begin{abstract}
Доказывается конечная базируемость произвольного $T$-пространства над полем нулевой характеристики. Этот результат обобщает результат Кемера о конечной базируемости любой системы ассоциативных тождеств над полем нулевой характеристики.

Библиография: 16 наименований.
\end{abstract}

\section{§1. Введение}

Эта работа посвящена доказательству аналога проблемы Шпехта для $T$-пространств. Пусть $F=\Phi\langle S\rangle$ - свободная счетно порожденная ассоциативная алгебра без единицы над коммутативным кольцом с единищей $\Phi . T$-nространством алгебры $F$ назовем любой $\Phi$-подмодуль $L$ в $F$, замкнутый относительно действия всех эндоморфизмов $\Phi$-алгебры $F$, т. е. подстановок вида $s \rightarrow f_{s}$, где $s \in S$ и $f_{s} \in F$. Если сушествует такое конечное множество многочленов $f_{1}, \ldots, f_{n}$ из $L$, что минимальное $T$-пространство, содержашее все $f_{i}$, совпадает с $L$, то $L$ называется конечно базируемым $T$-пространством. В случае, когда $L$ есть идеал, $T$-пространство $L$ называется $T$-идеалом. Легко проверить, что любое $T$-пространство, являющееся односторонним идеалом, есть двусторонний идеал, т. е. $T$-идеал. Примером $T$-пространства, не являющегося $T$-идеалом, является множество центральных полиномов. В этой статье мы рассматриваем в основном случай, когда $\Phi$ - поле нулевой характеристики.

Теория $T$-идеалов хорошо развита, и теорема Кемера [6] утверждает, что любой $T$-идеал над полем нулевой характеристики конечно базируем (как $T$-идеал). При этом техника А.Р. Кемера существенно использует структурную теорию и тот факт, что изучаемые объекты являются идеалами. Однако возникает подозрение, что требование замкнутости относительно умножения не является необходимым и можно доказывать конечную базируемость $T$-пространств. Впервые такие вопросы конечной базируемости изучались А. В. Гришиным в [8], [9]. Следует отметить, что в своих исследованиях Гришин ограничился локальным случаем, т. е. случаем, когда все многочлены зависят от конечного множества переменных. Однако А.Р. Кемер разработал технику $Z_{2}$-градуированных тождеств, используя теорию представления симметрической группы, и свел проблему Шпехта к локальному случаю, заменив $T$-идеалы на $T_{2}$-идеалы. $T_{2}$-идеалы - это идеалы свободной алгебры, замкнутые относительно некоторой подполугрупшы полугруппы всех подстановок. Вводим понятие $T_{2}$-пространства, аналогичное понятию $T_{2}$-идеала, 
и доказываем похожие локализационные утверждения. При этом приходится вводить понятие $T^{\prime}$-пространства, для которого полугруппа инвариантных подстановок, вообще говоря, у́же, чем полугруппа всех подстановок. Все результаты, описываюшие локализацию для $T$-пространств, изложены в $\S 2-4$.

В той части настоящей работы, где рассматривается локализованный случай, мы используем введенную в работах [8], [9] технику квазимногочленов. При этом используются различные фильтрации, символические степени, а также метод ширины, аналогично тому, как это делалось в работах [8], [9]. Детали некоторых доказательств $\S 6,8$ читатель может найти в вьшеупомянутых работах А.В. Гришина.

Другим важным соображением является лемма Артина-Рисса, способ применения которой к проблемам конечной базируемости был замечен А. Я. Беловым. Для согласования последнего подхода с методом ширины А. В. Гришина автор вводит понятие бесконечно погружающейся последовательности относительно фильтра-

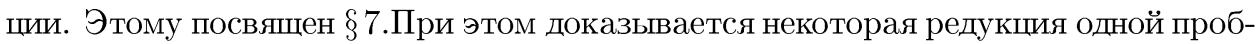
лемы А. В. Гришина для свободных алгебр над кольцом целых чисел. Выбранный подход позволяет в $\S 8$ быстро завершить доказательство следуюшего основного результата.

ТЕОрема. Любое T-пространство алгебры $K\langle X\rangle$, где $X$ - счетный алфавит и $K$ - поле нулевой характеристики, конечно базируемо.

Заметим, что полученное доказательство не использует теорему Кемера о конечной базируемости $T$-идеалов. Единственное место, где есть на нее ссылка, это лемма 2, но сама она в доказательстве не участвует.

В случае, когда характеристика основного поля положительна, ситуация с конечной базируемостью совсем другая. Как показал А.Р. Кемер в работе [7], в локальном случае над бесконечным полем любой $T$-идеал конечно базируем. Однако существуют бесконечно базируемые локальные $T$-пространства над любыми полями положительной характеристики. Соответствуюшие примеры построены автором в [4]. Там же строятся примеры бесконечно базируемых $T$-пространств, имеюших по каждой переменной степень $p$, над любыми полями характеристики $p>2$. До этого А.В. Гришиным [10] были построены аналогичные примеры в случае характеристики 2 . Примеры бесконечно базируемых $T$-идеалов построены А.Я. Беловым, а также автором и А. В. Гришиным в работах [10], [11], [5] и [16]. Таким образом, выше упомянутые работы демонстрируют связь и различие между понятиями $T$-пространства и $T$-идеала.

Автор благодарит В.Н. Латьшева, А.В. Гришина и А.Я. Белова за полезные обсуждения и внимание, проявленное к данной работе.

\section{§2. $T$-пространства, не содержащие ненулевых $T$-идеалов}

1. Общие соглашения. Пусть $K$-поле нулевой характеристики, $X=\left\{x_{1}, \ldots\right.$ $\left.\ldots, x_{n}, \ldots\right\}$ - основной счетный алфавит и $F=K\langle X\rangle-$ свободная ассоциативная алгебра без единицы. Через $T$ обозначим полугруппу эндоморфизмов $F$, а через $P$ обозначим множество полилинейных многочленов из $F$. 
Для однородного многочлена $f$ любой свободной ассоциативной алгебры через $\operatorname{vr}(f)$ обозначим множество переменных, от которых зависит $f$, т. е. множество переменных, по которым $f$ имеет ненулевую степень. В частности, $\operatorname{vr}(0)=\varnothing$. Пусть далее $P^{(n)}=\left\{f \in P: \operatorname{vr}(f)=\left\{x_{1}, \ldots, x_{n}\right\}\right\}$. Хорошо известно, что для нулевой характеристики основного поля любое $T$-пространство $L$ алгебры $F$ порождается пространствами $P^{(n)} \cap L, n \in \mathbb{N}$. Для двух слов $u$ и $v$ считаем $u \sim_{c} v$, если слова $u$ и $v$ получаются друг из друга циклическим сдвигом. Очевидно, $\sim_{c}$ есть отношение эквивалентности на словах. Везде ниже, в $\S 2-4$, через $S_{n}$ будем обозначать симметрическую группу степени $n$. При этом $S_{0}$ - единичная группа.

Введем одно соглашение, которое будет действовать до конца $§ 3$. Пусть встречается выражение

$$
\sum_{k=1}^{m} f_{k}\left(g_{k, 1}, \ldots, g_{k, n(k)}\right),
$$

о значении которого утверждается, что оно принадлежит некоторой свободной ассоциативной алгебре (не обязательно $F$ ), и $f_{k}$ - полилинейные многочлены. Обозначим значение данного выражения через $h$. Тогда мы считаем дополнительно, что для любого $k=1, \ldots, m$ многочлены $g_{k, 1}, \ldots, g_{k, n(k)}$ полилинейные, $\operatorname{vr}\left(g_{k, 1}\right)$, $\ldots, \operatorname{vr}\left(g_{k, n(k)}\right) \subset \operatorname{vr}(h)$, и если все многочлены $g_{k, 1}, \ldots, g_{k, n(k)}$ ненулевые, то $\operatorname{vr}\left(g_{k, 1}\right) \sqcup \cdots \sqcup \operatorname{vr}\left(g_{k, n(k)}\right)=\operatorname{vr}(h)$. Напомним, что выражение $A_{1} \sqcup \cdots \sqcup A_{n}=A$ означает $A_{1} \cup \cdots \cup A_{n}=A$ и $i \neq j \Longrightarrow A_{i} \cap A_{j}=\varnothing$.

2. Получение тождеств. Введем еще одно обозначение. Пусть $f \in P, x_{1} \in$ $\operatorname{vr}(f)$ и $g \in P^{(m)}$. Для $k \geqslant 2$ через $C_{k, m}(g)$ обозначим полином, получаюшийся из $g$ заменой $x_{i} \rightarrow x_{i+1+m(k-2)}, i=1, \ldots, m$. Пусть $A \subset X$. Через $C(f, g)$ и $C_{A}(f, g)$ обозначим полиномы, полученные из $f$ заменами $x_{i} \rightarrow C_{i, m}(g), i \in \operatorname{vr}(f) \backslash\left\{x_{1}\right\}$ и $x_{i} \rightarrow C_{i, m}(g), i \in \operatorname{vr}(f) \backslash\left(\left\{x_{1}\right\} \cup A\right)$ соответственно.

Ранее в [4] были доказаны следующие утверждения.

Лемма 1. Пусть $h=\sum_{i=1}^{k} g_{i} f g_{i}^{\prime} \in L \cap P$, әде $L$ - некоторое $T$-пространство алгебры $F$. Тогда $\sum_{i=1}^{k} g_{i}\left[t, x_{k}\right] g_{i}^{\prime} \in L$ для любой переменной $t \in X$.

СлеДСТВИЕ 1. Пусть $h=\sum_{i=1}^{k} g_{i} x_{l} f g_{i}^{\prime} \in L \cap P$, где $L$ - некоторое $T$-nространство алгебры $F$. Тогда $\sum_{i=1}^{k} g_{i} x_{l} f t g_{i}^{\prime} \in L$ для любой переменной $t \in X$.

Докажем сначала теорему, позволяющую сказать, содержит ли данное $T$-пространство ненулевой $T$-идеал. Через $L_{0}$ обозначим $T$-пространство, порожденное коммутатором $\left[x_{1}, x_{2}\right]$, и пусть $L_{0}^{(n)}=P^{(n)} \cap L_{0}$. Имеет место

Tеорема 1. Пусть $L$ - некоторое T-пространство алгебры F. Следующие условия әквивалентны:

a) $L$ не содержит ненулевых $T$-идеалов;

b) $L \subset L_{0}$.

ДоКАЗАТЕЛЬСТво. b) $\Longrightarrow$ a). Предположим, что существует ненулевой $T$-идеал $I \subset L \subset L_{0}$. Пусть $0 \neq f \in I$. Выберем $x_{k} \notin \operatorname{vr}(f)$. Пусть $f=\sum_{i=1}^{m} \alpha_{i} u_{i}$, где $u_{1}, \ldots, u_{m}-$ попарно различные слова и $\alpha_{i} \in K$. Имеем

$$
x_{k} f=\sum_{i=1}^{m} \alpha_{i} x_{k} u_{i} \in I \subset L_{0} .
$$


В этом представлении различные слова $x_{k} u_{i}$ лежат в различных классах эквивалентности относительно $\sim_{c}$ и, следовательно, все $\alpha_{i}$ равны нулю. Отсюда получаем $f=0$. Противоречие.

a) $\Longrightarrow$ b). Предположим, что $L$ не подмножество $L_{0}$. Тогда существует однородный многочлен $f \in L \backslash L_{0}$. Можно считать, что $\operatorname{vr}(f)=\left\{x_{1}, \ldots, x_{m}\right\}$ и $\operatorname{deg}_{x_{i}} f=n_{i}, i=1, \ldots, m$. Пусть $f^{\prime}$ - полная линеаризация многочлена $f$. Если получается, что $f^{\prime} \in L_{0}$, то, подставляя вместо каждой переменной этого многочлена ту переменную $x_{i}$, из которой она получилась в процессе линеаризации, получим $n_{1} ! \ldots n_{k} ! f \in L_{0}$. В силу того, что char $K=0$, имеем $f \in L_{0}$, что не так. Таким образом, можно считать, что $f^{\prime} \in\left(L \cap P^{(n)}\right) \backslash L_{0}$ для некоторого $n$.

В силу определения полинома $C(f, g)$ получаем, что $C\left(f^{\prime}, f^{\prime}\right)$ является линейной комбинацией полиномов вида

$$
C_{i_{1}, n}\left(f^{\prime}\right) \ldots C_{i_{k-1}, n}\left(f^{\prime}\right) x_{1} C_{i_{k+1}, n}\left(f^{\prime}\right) \ldots C_{i_{n}, n}\left(f^{\prime}\right)
$$

где $\left\{i_{1}, \ldots, i_{k-1}, i_{k+1}, \ldots, i_{n}, 1\right\}=\{1, \ldots, n\}$.

Так как $C_{i_{l}, n}\left(f^{\prime}\right) \in L$, то по лемме 1 имеем $\left[C_{i_{l}, n}\left(f^{\prime}\right), t\right] \in L$ для любой переменной $t \in X$. Следовательно,

$$
\begin{aligned}
& C_{i_{1}, n}\left(f^{\prime}\right) \ldots C_{i_{k-1}, n}\left(f^{\prime}\right) x_{1} C_{i_{k+1}, n}\left(f^{\prime}\right) \ldots C_{i_{n}, n}\left(f^{\prime}\right) \\
& \quad=C_{i_{2}, n}\left(f^{\prime}\right) \ldots C_{i_{k-1}, n}\left(f^{\prime}\right) x_{1} C_{i_{k+1}, n}\left(f^{\prime}\right) \ldots C_{i_{n}, n}\left(f^{\prime}\right) C_{i_{1}, n}\left(f^{\prime}\right)(\bmod L) .
\end{aligned}
$$

Здесь надо выполнить подстановку $t \rightarrow C_{i_{2}, n}\left(f^{\prime}\right) \ldots C_{i_{k-1}, n}\left(f^{\prime}\right) x_{1} C_{i_{k+1}, n}\left(f^{\prime}\right) \ldots$ $\ldots C_{i_{n}, n}\left(f^{\prime}\right)$. Продолжая процесс переброски вправо выражений $C_{i_{j}, n}\left(f^{\prime}\right)$, стояших перед $x_{1}$, получаем

$$
\begin{aligned}
& C_{i_{1}, n}\left(f^{\prime}\right) \ldots C_{i_{k-1}, n}\left(f^{\prime}\right) x_{1} C_{i_{k+1}, n}\left(f^{\prime}\right) \ldots C_{i_{n}, n}\left(f^{\prime}\right) \\
& \quad=x_{1} C_{i_{k+1}, n}\left(f^{\prime}\right) \ldots C_{i_{n}, n}\left(f^{\prime}\right) C_{i_{1}, n}\left(f^{\prime}\right) \ldots C_{i_{k-1}, n}\left(f^{\prime}\right)(\bmod L)
\end{aligned}
$$

Таким образом, $C\left(f^{\prime}, f^{\prime}\right)=x_{1} h(\bmod L)$ для соответствующего $h$. Так как $f^{\prime} \notin L_{0}$, то $h \neq 0$. В силу следствия 1 имеем $x_{1} h g \in L$ для любого многочлена $g$ из $F$. Поэтому $T$-идеал, порожденный многочленом $x_{1} h$, содержится в $L$. Теорема доказана.

Для $T$-идеала $I$ через $[I, F]$ обозначим подпространство алгебры $F$, состояшие из линейных комбинаций коммутаторов вида $[f, h]$, где $f \in I$ и $h \in F$. Легко проверить, что $[I, F]$ есть $T$-пространство. Имеет место

Теорема 2. Пусть $L$ - ненулевое $T$-пространство. Тогда $[I, F] \subset L$ для некоторого ненулевого $T$-идеала $I$. 
ДокАЗАТЕЛЬСтво. Если $L$ не содержится в $L_{0}$, то утверждение следует из теоремы 1 . Считаем теперь, что $L \subset L_{0}$. Пусть $f$-ненулевой многочлен из $L \cap P^{(n)}$. Тогда справедливо представление $f=\sum_{i=2}^{n}\left[f_{i}, x_{i}\right]$, так как множество многочленов $\left\{\left[g, x_{i}\right]: i=2, \ldots, n\right.$ и $\left.\operatorname{vr}(g)=\left\{x_{1}, \ldots, x_{i-1}, x_{i+1}, \ldots, x_{n}\right\}\right\}$ порождает $L_{0} \cap P_{n}$ как линейное пространство. Так как $f \neq 0$, то сушествует $i_{0} \geqslant 2$ такое, что $f_{i_{0}} \neq 0$. Пусть $f^{\prime}$-полином, полученный из $f$ заменой $x_{i_{0}}$ на $x_{l} x_{i_{0}}$ для произвольного числа $l \geqslant n(n-1)+2$. Тогда имеем

$$
f^{\prime}=\sum_{\substack{i=2 \\ i \neq i_{0}}}^{n}\left[f_{i}^{\prime}, x_{i}\right]+\left[f_{i_{0}}, x_{l} x_{i_{0}}\right]=\sum_{\substack{i=2 \\ i \neq i_{0}}}^{n}\left[f_{i}^{\prime}, x_{i}\right]+\left[f_{i_{0}} x_{l}, x_{i_{0}}\right]+\left[x_{i_{0}} f_{i_{0}}, x_{l}\right] .
$$

Так как $f_{i_{0}} \neq 0$ и $x_{i_{0}} \notin \operatorname{vr}\left(f_{i_{0}}\right)$, то $x_{i_{0}} f_{i_{0}} \notin L_{0}$. По лемме 1 имеем

$$
C_{\left\{x_{l}\right\}}\left(f^{\prime}, f\right)=\left[C_{i_{0}, n}(f) C\left(f_{i_{0}}, f\right), x_{l}\right] \quad(\bmod L) .
$$

Пусть $f_{i_{0}}=\sum_{\sigma \in S^{\prime}} \alpha_{\sigma} x_{\sigma(1)} \ldots x_{\sigma(n-1)}$, где $S^{\prime}$ - множество биекций из $\{1, \ldots$ $\ldots, n-1\}$ в $\left\{1, \ldots, i_{0}-1, i_{0}+1, \ldots, n\right\}$. Положим $n(\sigma)=\sigma^{-1}(1), \sigma \in S^{\prime}$. Фактически $n(\sigma)$ равно номеру слева позиции в мономе $x_{\sigma(1)} \ldots x_{\sigma(n-1)}$, на которой стоит $x_{1}$. В силу леммы 1 имеем $\left[f, x_{l}\right] \in L$. Еще раз применяя лемму 1, получаем $\left[[f, t], x_{l}\right] \in L$ для любой переменной $t \in X$.

Отсюда, перенося вправо выражения вида $C_{i_{j}, n}(f)$, стоящие перед $x_{1}$, аналогично тому, как мы это делали при доказательстве теоремы 1 , получаем

$$
\begin{aligned}
& {\left[C_{i_{0}, n}(f) C_{\sigma(1), n}(f) \ldots C_{\sigma(n(\sigma)-1), n}(f) x_{1} C_{\sigma(n(\sigma)+1), n}(f) \ldots C_{\sigma(n-1), n}(f), x_{l}\right]} \\
& \quad=\left[x_{1} h_{\sigma}, x_{l}\right](\bmod L)
\end{aligned}
$$

где $h_{\sigma}=C_{\sigma(n(\sigma)+1), n}(f) \ldots C_{\sigma(n-1), n}(f) C_{i_{0}, n}(f) C_{\sigma(1), n}(f) \ldots C_{\sigma(n(\sigma)-1), n}(f)$. Тогда $\left[x_{1} g, x_{l}\right] \in L$, где $g=\sum_{\sigma \in S^{\prime}} \alpha_{\sigma} h_{\sigma}$. Так как $x_{i_{0}} f_{i_{0}} \notin L_{0}$, то $g \neq 0$.

По следствию 1 получаем $[I, F] \subset L$, где $I$ есть $T$-идеал, порожденный многочленом $x_{1} g$. Теорема доказана.

Заметим, что справедлив следующий результат, которьй есть непосредственное следствие теоремы Кемера.

Лемма 2. Любая возрастающая чепочка T-пространств $L_{0} \subset L_{1} \subset \ldots$ $\cdots \subset L_{i} \subset \cdots \subset F$ стабилизируется.

ДокАЗАТЕЛЬСТво. Если $f \in L_{i} \cap P^{(n)}$, то $f=x_{1} g\left(\bmod L_{0}\right)$ для некоторого $g$. Через $L_{i}^{\prime}$ обозначим $T$-пространство, порожденное всеми такими многочленами $x_{1} g$, для всевозможных $f \in L_{i} \cap P^{(n)}$ и $n \in \mathbb{N}$. Легко заметить, что любое $L_{i}^{\prime}$ есть $T$-идеал. Тогда цепочка $L_{1}^{\prime} \subset \cdots \subset L_{i}^{\prime} \subset \cdots \subset F$ стабилизируется по теореме Кемера [6], т. е. $L_{r}^{\prime}=L_{s}^{\prime}$ при $r \geqslant s$ для некоторого фиксированного $s$. Пусть теперь $f \in L_{r} \cap P^{(n)}$ и $f=x_{1} g\left(\bmod L_{0}\right)$. Тогда $x_{1} g \in L_{s}^{\prime}$, и так как $L_{s}^{\prime} \subset L_{s}$, то имеем $x_{1} g \in L_{s}$. Так как $L_{0} \subset L_{s}$, то $f \in L_{s}$ и $L_{r}=L_{s}$. Лемма доказана. 
3. $T^{\prime}$-пространства. Пусть $Y=\left\{y_{1}, \ldots, y_{n}, \ldots\right\}$-еше одно множество переменных и $z_{0}-$ новая переменная. При этом мы считаем, что $X \cap Y=\varnothing$ и $z_{0} \notin X \cup Y$. Положим $F^{\prime}=K\langle X \cup Y\rangle$ и $F^{\prime \prime}=K\left\langle X \cup Y \cup\left\{z_{0}\right\}\right\rangle$. Тогда $F \subset F^{\prime} \subset F^{\prime \prime}$. Аналогично тому, как это было сделано для алгебры $F$, вводятся множество полилинейных многочленов $P^{\prime}$ и $P^{\prime \prime}$ алгебр $F^{\prime}$ и $F^{\prime \prime}$ соответственно и множество переменных $\operatorname{vr}(f)$, от которых зависит однородньй многочлен $f$ из $F^{\prime}$ или $F^{\prime \prime}$.

Линейное пространство $L \subset F^{\prime}$ назовем $T^{\prime}$-пространством, если $\varphi(L) \subset L$ для любого эндоморфизма $\varphi$ алгебры $F^{\prime}$ такого, что $\varphi(F) \subset F$. Полугруппу таких эндоморфизмов алгебры $F^{\prime}$ обозначим через $H^{\prime}$. Для изучения порождающих $T$-пространств, содержашихся в $L_{0}$, введем понятие присоединенного $T^{\prime}$-пространства.

До конца $\S 3$ для любого $T$-пространства $L$ алгебры $F$ через $L^{\prime \prime}$ обозначаем $T$-пространство алгебры $F^{\prime \prime}$, порожденное $L$. Очевидно, $L^{\prime \prime} \cap F=L$.

Пусть теперь $L \subset L_{0}$. Присоединенное $T^{\prime}$-пространство $A(L)$ для $T$-пространства $L$ породим как $T^{\prime}$-пространство многочленами $f \in P^{\prime}$ такими, что

$$
\left[f, z_{0}\right]+\sum_{k=1}^{m}\left[f_{k}, x_{i_{k}}\right] \in L^{\prime \prime} \cap P^{\prime \prime}
$$

для некоторых $f_{k}$ и $i_{k}$. Множество таких многочленов $f$ обозначим через $B(L)$.

Лemma 3. $B(L)=A(L) \cap P^{\prime}$.

ДокАЗАТЕЛЬСтво. Неформально говоря, требуется показать, что любое полилинейное $T^{\prime}$-следствие из $B(L)$ лежит в $B(L)$.

Пусть $f$ и $g$-ненулевые многочлены из $B(L), \operatorname{vr}(f)=\operatorname{vr}(g)$ и $\alpha, \beta \in K$. Имеем $\left[f, z_{0}\right]+\sum_{k=1}^{m}\left[f_{k}, x_{i_{k}}\right] \in L^{\prime \prime} \cap P^{\prime \prime}$ и $\left[g, z_{0}\right]+\sum_{k=1}^{l}\left[g_{k}, x_{j_{k}}\right] \in L^{\prime \prime} \cap P^{\prime \prime}$ для некоторых $f_{i}, g_{j} \in P^{\prime \prime}$. Тогда

$$
\left[\alpha f+\beta g, z_{0}\right]+\sum_{k=1}^{m}\left[\alpha f_{k}, x_{i_{k}}\right]+\sum_{k=1}^{m}\left[\beta g_{k}, x_{j_{k}}\right] \in L^{\prime \prime} \cap P^{\prime \prime} .
$$

Этим доказано, что $\alpha f+\beta g \in B(L)$.

Пусть теперь $f$-ненулевой многочлен из $B(L), \varphi \in H^{\prime}$ и $\varphi(f) \in P^{\prime}$. Достаточно считать, что $\varphi\left(x_{i}\right)=u_{i}, \varphi\left(y_{i}\right)=v_{i}$, где $u_{i}, v_{i}$ - полилинейные слова и множества переменных $\operatorname{vr}\left(u_{i}\right), \operatorname{vr}\left(v_{j}\right)$ попарно не пересекаются. По определению $H^{\prime}$ имеем $u_{i} \in F$.

Так как $f \in B(L)$, то имеем $\left[f, z_{0}\right]+\sum_{k=1}^{m}\left[f_{k}, x_{i_{k}}\right] \in L^{\prime \prime} \cap P^{\prime \prime}$ для некоторых $f_{k} \in P^{\prime \prime}$. Определим эндоморфизм $\psi$ алгебры $F^{\prime \prime}$ по правилу: $\psi\left(z_{0}\right)=z_{0}$ и $\psi(t)=$ $\varphi(t)$ для любой переменной $t \in X \cup Y$. Тогда

$$
\psi\left(\left[f, z_{0}\right]+\sum_{k=1}^{m}\left[f_{k}, x_{i_{k}}\right]\right)=\left[\varphi(f), z_{0}\right]+\sum_{k=1}^{m}\left[\psi\left(f_{k}\right), u_{i_{k}}\right] \in L^{\prime \prime} \cap P^{\prime \prime} .
$$

Учитьвая формулу

$$
\left[y, x_{1} \ldots x_{n}\right]=\left[y x_{1} \ldots x_{n-1}, x_{n}\right]+\left[x_{n} y x_{1} \ldots x_{n-2}, x_{n-1}\right]+\cdots+\left[x_{2} \ldots x_{n} y, x_{1}\right],
$$

представим каждый полином $\left[\psi\left(f_{k}\right), u_{i_{k}}\right]$ в виде суммы полиномов вида $\left[h, x_{i}\right]$. Отсюда получаем $\varphi(f) \in B(L)$. Лемма доказана.

Докажем теперь теорему, связываюшую порождающие $L$ и $A(L)$. 
Теорема 3. Пусть $\left\{g_{j}: j \in \Lambda\right\}$ - ненулевые полилинейные многочлены из $F^{\prime}$, порождающие $A(L)$ как $T^{\prime}$-пространство. Пусть также

$$
h_{j}=\left[g_{j}, z_{0}\right]+\sum_{k=1}^{m_{j}}\left[g_{j, k}, x_{l(j, k)}\right] \in L^{\prime \prime} \cap P^{\prime \prime}
$$

$u \psi_{j}: F^{\prime \prime} \rightarrow F, \quad j \in \Lambda,-$ гомоморфизмы, переводящие переменные из $X \cup Z \cup$ $\left\{z_{0}\right\}$ в переменные из $X$, такие, что $\left.\psi_{j}\right|_{X}$ есть тождественное отображение $X$ в себя и $\left.\psi_{j}\right|_{\operatorname{vr}\left(h_{j}\right)}$ инбективно.

Тогда полиномы $\left\{\psi_{j}\left(h_{j}\right): j \in \Lambda\right\}$ порождают $L$ как T-пространство.

ДоказАТЕЛЬство. Через $M$ обозначим $T$-пространство алгебры $F$, порожденное полиномами $\left\{\psi_{j}\left(h_{j}\right): j \in \Lambda\right\}$. Все эти полиномы принадлежат $L$, и, следовательно, $M \subset L$. Отсюда $A(M) \subset A(L)$. С другой стороны, все $h_{j}$ принадлежат $M^{\prime \prime} \cap P^{\prime \prime}$. Поэтому $g_{j} \in A(M)$. По условию леммы тогда $A(M)=A(L)$.

Предположим, что $M \neq L$. Тогда существует такое $n \geqslant 1$, для которого выполнено $\left(L \cap P^{(n)}\right) \backslash M \neq \varnothing$. Положим

$$
i_{0}=\max \left\{i_{0}^{\prime}: \text { сушествует } \sum_{k=0}^{m^{\prime}}\left[f_{k}^{\prime}, x_{i_{k}^{\prime}}\right] \in\left(L \cap P^{(n)}\right) \backslash M \text { и } i_{0}^{\prime}<\cdots<i_{m^{\prime}}^{\prime}\right\} \text {. }
$$

Пусть $f \in\left(L \cap P^{(n)}\right) \backslash M$ и $f=\sum_{k=0}^{m}\left[f_{k}, x_{i_{k}}\right]$ для некоторых $i_{k}$ таких, что $i_{0}<i_{1}<\cdots<i_{m}$. Из условия (1) следует, что $f_{0} \neq 0$.

Пусть $\psi: F \rightarrow F^{\prime \prime}$ - гомоморфизм такой, что $\psi\left(x_{i}\right)=y_{i}$ при $i=1, \ldots, i_{0}-1$, $\psi\left(x_{i_{0}}\right)=z_{0}$ и $\psi\left(x_{i}\right)=x_{i}$ при $i=i_{0}+1, \ldots, n$. Тогда

$$
\psi(f)=\left[\psi\left(f_{0}\right), z_{0}\right]+\sum_{k=1}^{m}\left[\psi\left(f_{k}\right), x_{i_{k}}\right] \in L^{\prime \prime} \cap P^{\prime \prime}
$$

Отсюда $\psi\left(f_{0}\right) \in B(L) \subset A(L)=A(M)$. Следовательно, $\psi\left(f_{0}\right) \in A(M) \cap P^{\prime}$. По лемме 3 имеем $A(M) \cap P^{\prime}=B(M)$. Отсюда $\psi\left(f_{0}\right) \in B(M)$. По определению операции $B$ имеем

$$
h=\left[\psi\left(f_{0}\right), z_{0}\right]+\sum_{k=1}^{m_{0}}\left[g_{k}, x_{j_{k}}\right] \in M^{\prime \prime} \cap P^{\prime \prime} .
$$

Если предположить, что для некоторого $k=1, \ldots, m_{0}$ выполнено $j_{k} \leqslant i_{0}$, то в силу соглашения, сформулированного в начале этого параграфа, $j_{k} \in \operatorname{vr}(h)$. Так как $\psi\left(f_{0}\right) \neq 0$, то в силу того же соглашения $\psi\left(f_{0}\right)$ зависит от переменной $x_{j_{k}}$. Так как $j_{k} \leqslant i_{0}$, то приходим к противоречию со сделанным предположением. Поэтому для любого $k$ имеем $j_{k}>i_{0}$. Пусть $\psi^{\prime}: F^{\prime \prime} \rightarrow F$ - гомоморфизм такой, что $\psi^{\prime}\left(y_{i}\right)=x_{i}$ при $i=1, \ldots, i_{0}-1, \psi^{\prime}\left(z_{0}\right)=x_{i_{0}}$ и $\psi^{\prime}\left(x_{i}\right)=x_{i}$ при $i=i_{0}+1, \ldots, n$. Такой 
гомоморфизм $\psi^{\prime}$ связан с гомоморфизмом $\psi$ соотношением $\left.\left(\psi^{\prime} \psi\right)\right|_{P^{(n)}}=\mathrm{id}_{P^{(n)}}$. Имеем

$$
\begin{gathered}
\psi^{\prime}(h)=\left[f_{0}, x_{i_{0}}\right]+\sum_{k=1}^{m_{0}}\left[\psi^{\prime}\left(g_{k}\right), x_{j_{k}}\right] \in M \cap P, \\
f-\psi^{\prime}(h)=\sum_{k=1}^{m}\left[f_{k}, x_{i_{k}}\right]-\sum_{k=1}^{m_{0}}\left[\psi^{\prime}\left(g_{k}\right), x_{j_{k}}\right] \in L \cap P^{(n)} .
\end{gathered}
$$

Согласно выбору $i_{0}$ получаем $f-\psi^{\prime}(h) \in M$. Но $\psi^{\prime}(h) \in M$, отсюда $f \in M$. Противоречие. Теорема доказана.

\section{§3. Супероризация для $T$-пространств}

\section{1. Основные факты теории представлений симметрической груп-} пы. В настоящей работе тензорное произведение $\otimes$ понимается в смысле $\otimes_{K}$, т. е. над основным полем $K$. Набор целых чисел $\left(l_{1}, \ldots, l_{s}\right)$ называется разбиением иисла $l$, если $l_{1} \geqslant \cdots \geqslant l_{s} \geqslant 1$ и $\sum_{i=1}^{s} l_{i}=l$. Пусть $D$ - таблища Юнга, соответствующая разбиению $\left(l_{1}, \ldots, l_{s}\right)\left(l_{i}\right.$ - длина $i$-ой строки). Любому заполнению клеток этой таблищы неповторяюшимися числами от 1 до $l$ соответствует диаграмма Юнга $C$. В данном случае пишем $D=\operatorname{tab}(C)$. Обозначим через $|D|$ и $|C|$ количество клеток в таблище $D$ и в диаграмме $C$. В наших обозначения это число равно $l$. Через $D^{*}$ обозначим диаграмму Юнга, в $i$-ой строке которой слева направо стоят числа $\sum_{j=1}^{i-1} l_{j}+1, \ldots, \sum_{j=1}^{i} l_{j}$. Группа $P_{C}\left(\right.$ соответственно $\left.Q_{C}\right)$ состоит из всех подстановок, оставляющих инвариантными все строки (столбцы) диаграммы $C$. Пусть

$$
e_{C}=\sum_{\substack{p \in P_{C} \\ q \in Q_{C}}}(\operatorname{sgn} q) p q
$$

- симметризатор Юнга. Левый идеал алгебры $K S_{l}$, порожденный элементом $e_{C}$, обозначим через $J_{C}$, т.е. $J_{C}=K S_{l} e_{C}$. Более подробно теория представлений симметрической группы изложена в [12].

В дальнейшем будем использовать следующие факты об алгебре $K S_{n} \otimes K S_{m}$ :

1) кольца $K S_{n} \otimes K S_{m}$ и $K\left(S_{n} \times S_{m}\right)$ изоморфны и левый $\left(K S_{n} \otimes K S_{m}\right)$-модуль $K S_{n} \otimes K S_{m}$ вполне приводим;

2 ) левый идеал алгебры $K S_{n} \otimes K S_{m}$, порожденный элементом $e_{C_{1}} \otimes e_{C_{2}}$, обозначим через $J_{C_{1}, C_{2}}$, тогда $J_{C_{1}, C_{2}}=J_{C_{1}} \otimes J_{C_{2}}$.

Справедлива

ЛЕмма 4 (о представлениях). Левые идеаль $J_{C_{1}, C_{2}}$ являются минимальHbl.Mu.

ДокаЗАТЕльство. Имеем $e_{C_{1}} \otimes e_{C_{2}} \neq 0$ и идеал $J_{C_{1}, C_{2}}$ ненулевой. Пусть $f-$ ненулевой элемент из $J_{C_{1}, C_{2}}$. Возможно представление $f=a_{1} \otimes b_{1}+\cdots+a_{s} \otimes b_{s}$, где элементы $b_{1}, \ldots, b_{s}$ линейно независимы над $K$, а элементы $a_{1}, \ldots, a_{s}$ не равны нулю. Так как $J_{C_{2}}$ - неприводимый $K S_{m}$-модуль и любой его эндоморфизм есть умножение на элемент поля $K$, то по теореме плотности [13] существует такой $r \in$ 
$K S_{m}$, что $r b_{1}=e_{C_{1}}, r b_{2}=0, \ldots, r b_{s}=0$. Тогда $(1 \otimes r) f=a_{1} \otimes e_{C_{2}} \neq 0$. В силу минимальности идеалов $J_{C_{1}}$ и $J_{C_{2}}$ элемент $a_{1} \otimes e_{C_{2}}$ порождает весь идеал $J_{C_{1}, C_{2}}$. Лемма доказана.

2. Основное утверждение. Пусть $\gamma=\left(i_{1}, \ldots, i_{k}\right)$, где каждое $i_{l}$ есть либо 0 , либо 1. Пусть $|\gamma|=k$ - длина набора $|\gamma|,|\gamma|_{X}$ - число нулей в наборе $\gamma$ и $|\gamma|_{Y}$ число единиц в наборе $\gamma$.

Пусть $n$ и $m$ - натуральные числа, не равные одновременно нулю, $\gamma=\left(i_{1}, \ldots\right.$ $\left.\ldots, i_{n+m}\right), n=|\gamma|_{X}$ и $m=|\gamma|_{Y}$. Через $P(\gamma)$ обозначим подпространство алгебры $F^{\prime}$, натянутое на полилинейные слова $u$ такие, что:

1) $\operatorname{vr}(u)=\left\{x_{1}, \ldots, x_{n}, y_{1}, \ldots, y_{m}\right\}$;

2 ) в слове $u$ на $l$-ой позиции слева стоит переменная из $X$, если $i_{l}=0$, и переменная из $Y$, если $i_{l}=1$.

Если в данном определении опустить условие 2), то получим определение пространства $P(n, m)$. Пространство $P(n, m)$ является $\left(K S_{n} \otimes K S_{m}\right)$-модулем. При этом элемент $\sigma_{1} \otimes \sigma_{2}$, где $\sigma_{1} \in S_{n}, \sigma_{2} \in S_{m}$, действует на произвольный многочлен $f \in P(n, m)$ по формуле $t\left(\sigma_{1} \otimes \sigma_{2}\right) f$, где $t\left(\sigma_{1} \otimes \sigma_{2}\right)$ - эндоморфизм алгебры $F^{\prime}$ такой, что

$$
t\left(\sigma_{1} \otimes \sigma_{2}\right)\left(x_{i}\right)=x_{\sigma_{1}(i)}, \quad i=1, \ldots, n, \quad t\left(\sigma_{1} \otimes \sigma_{2}\right)\left(y_{j}\right)=y_{\sigma_{2}(j)}, \quad j=1, \ldots, m .
$$

Очевидно, что $P(n, m)=\bigoplus\left\{P(\gamma):|\gamma|_{X}=n,|\gamma|_{Y}=m\right\}$ и любое пространство $P(\gamma)$ является $\left(K S_{n} \otimes K S_{m}\right)$-подмодулем модуля $P(n, m)$.

Введем на множестве переменных $X \cup Y \cup\left\{z_{0}\right\}$ порядок следующим образом: $x_{i}<x_{j}$ при $i<j, y_{i}<y_{j}$ при $i<j, x_{i}<y_{j}, z_{0}<x_{i}, z_{0}<y_{i}$.

Выражения max и min, примененные к множествам переменных из $X \cup Y \cup$ $\left\{z_{0}\right\}$, будем далее понимать именно в смысле введенного порядка $<$. Далее в этом параграфе считаем, что $\min (\varnothing)=\max (\varnothing)=z_{0}$.

Пусть $f \in P^{\prime \prime}$. Предположим, что $A=\left\{t_{1}, \ldots, t_{k}\right\}$ - непустое подмножество множества $\operatorname{vr}(f)$ и $t_{1}<\cdots<t_{k}$. Для $\sigma \in S_{k}$ определим гомоморфизм $p_{A}(\sigma): F^{\prime} \rightarrow$ $F^{\prime}$ по формулам $p_{A}(\sigma)(t)=t$, если $t \in\left(X \cup Y \cup\left\{z_{0}\right\}\right) \backslash A$, и $p_{A}(\sigma)\left(t_{i}\right)=t_{\sigma(i)}$, $i=1, \ldots, k$. Положим

$$
\begin{aligned}
S_{A} f & =\sum_{\sigma \in S_{k}} p_{A}(\sigma)(f), \\
\Lambda_{A} f & =\sum_{\sigma \in S_{k}}(\operatorname{sgn} \sigma) p_{A}(\sigma)(f) .
\end{aligned}
$$

Если $A=\varnothing$, то считаем $S_{A} f=\Lambda_{A} f=f$.

Используя технику работы [2], докажем следующую лемму.

Лемма 5. Пусть L и Г - ненулевые T-пространства алгебры $F$ такие, что $L \subset \Gamma \subset L_{0}$. Тогда существулт числа $r$ u $t$, зависящие только от $L$, обладающие следующим свойством: если $f \in L_{0} \cap P$ и для любого разбиения

$$
A_{1} \sqcup \cdots \sqcup A_{r} \sqcup B_{1} \sqcup \cdots \sqcup B_{t}=\operatorname{vr}(f)
$$

выполнено $S_{A_{1}} \ldots S_{A_{r}} \Lambda_{B_{1}} \ldots \Lambda_{B_{t}} f \in \Gamma$, mо $f \in \Gamma$. 
ЗАмечАниЕ. Если $L \not \subset L_{0}$, то по теореме 1 существует ненулевой $T$-идеал $\Gamma \subset L$. Предложение 1 из работы [2] фактически означает справедливость аналога данной леммы для $T$-пространства $L$ такого, что $L \not \subset L_{0}$. Это следует из того, что в этом случае имеется оценка для коразмерностей $\operatorname{dim}\left(P^{(n)} \mid L \cap P^{(n)}\right) \leqslant d^{n}$ при некотором $d$.

ДокАЗАТЕЛЬСТво. По теореме $2 T^{\prime}$-пространство $A(\Gamma)$ содержит некоторый ненулевой полилинейньй многочлен вида $y_{1} g_{0} y_{2}$, где $g_{0}$ зависит только от переменных из $Y$. Это значит, что $A(L)$ содержит ненулевой $T$-идеал алгебры $F^{\prime}$, порожденный многочленом $y_{1} g_{0} y_{2}$. Из теоремы Регева тогда следует, что существует $d \geqslant 1$ такое, что

$$
\operatorname{dim}(P(n, m) \mid A(L) \cap P(n, m)) \leqslant d^{n+m} .
$$

Пусть $\Gamma$-некоторое $T$-пространство, удовлетворяющее условию леммы. Тогда так как $L \subset \Gamma$, то $\operatorname{dim}(P(n, m) \mid A(\Gamma) \cap P(n, m)) \leqslant d^{n+m}$.

Для таблищы $D$, содержащей $l$ клеток, определим однородную компоненту

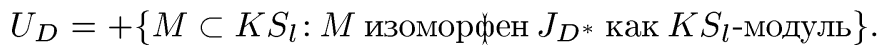

Тогда $U_{D}=+\left\{J_{C}: D=\operatorname{tab}(C)\right\}$ и $K S_{l}=\bigoplus\left\{U_{D}:|D|=l\right\}$.

Для таблиц $D_{1}$ и $D_{2}$, содержащих соответственно $n$ и $m$ клеток, положим

$$
U_{D_{1}, D_{2}}=+\left\{J_{C_{1}, C_{2}}: D=\operatorname{tab}\left(C_{1}\right), D=\operatorname{tab}\left(C_{2}\right)\right\}
$$

Очевидно, $U_{D_{1}, D_{2}}=U_{D_{1}} \otimes U_{D_{2}}$ и $K S_{n} \otimes K S_{m}=\bigoplus\left\{U_{D_{1}, D_{2}}:\left|D_{1}\right|=n,\left|D_{2}\right|=m\right\}$.

Пусть $M$ - некоторый подмодуль модуля $K S_{n} \otimes K S_{m}$ и $\gamma=\left(i_{1}, \ldots, i_{n+m}\right)-$ последовательность нулей и единиц такая, что $|\gamma|_{X}=n$ и $|\gamma|_{Y}=m$. Через $e(\gamma)$ обозначим моном из $P(\gamma)$, у которого на $l$-ом слева месте стоит $x_{j}$, если $i_{l}$ является $j$-ым слева нулем в $\gamma$, или $y_{k}$, если $i_{l}$ является $k$-ой слева единицей в $\gamma$. Положим $M(\gamma)=M e(\gamma)$. При этом если $M-$ минимальньй подмодуль, то $M(\gamma)-$ минимальный подмодуль модуля $P(n, m)$. Вообще-то, все минимальные подмодули модуля $P(n, m)$ не исчерпьваются модулями $M(\gamma)$.

Пусть $U_{D}^{(X)}=\left(U_{D} \otimes K S_{0}\right)\left(\gamma_{l}^{(0)}\right)$ и $U_{D}^{(Y)}=\left(K S_{0} \otimes U_{D}\right)\left(\gamma_{l}^{(1)}\right)$, где $l=|D|, \gamma_{l}^{(0)}-$ последовательность из $l$ нулей и $\gamma_{l}^{(1)}-$ последовательность из $l$ единиц. Таким образом построенные пространства $U_{D}^{(X)}$ и $U_{D}^{(Y)}$ являются каноническими реализациями модуля $U_{D}$ в пространствах $P(l, 0)$ и $P(0, l)$ соответственно.

При таких определениях получаем $U_{D_{1}, D_{2}}(\gamma)=U_{D_{1}, D_{2}} P(\gamma)$ в силу того, что $U_{D_{1}, D_{2}}-$ двусторонний идеал, и $U_{D_{1}, D_{2}} P(n, m)=\bigoplus\left\{U_{D_{1}, D_{2}}(\gamma):|\gamma|_{X}=n\right.$, $\left.|\gamma|_{Y}=m\right\}$.

Если $p, q$-натуральные числа, то через $D(p, q)$ обозначим таблицу Юнга, состоящую из $p$ равных столбцов длины $q$. Положим $b_{p, q}=\operatorname{dim} J_{D(p, q)^{*}}$. Из формулы крюков для размерности неприводимых модулей следует, что

$$
b_{p, q}=\frac{(p q) !}{(q !)^{p} r_{p}(q)}
$$


где $r_{p}(q)$ - многочлен с рациональными коэффициентами от $q$, степень и коэффициенты которого зависят только от $p$. Отсюда, воспользовавшись формулой Стирлинга, нетрудно получить, что

$$
\lim _{q \rightarrow \infty} \frac{b_{p, q}}{(p-1)^{p q}}=\infty
$$

Положим $t_{0}=d^{2}$, а число $r_{0}$ возьмем таким, чтобы выполнялось неравенство

$$
b_{t_{0}+1, r_{0}+1}>d^{2\left(t_{0}+1\right)\left(r_{0}+1\right)+1}
$$

что возможно в силу формулы (2). Положим $m_{0}=\left(t_{0}+1\right)\left(r_{0}+1\right), r=2 r_{0}+1$ и $t=2 t_{0}$. Выбор этих чисел $r$ и $t$ зависит только от числа $d$, которое определяется по $T$-пространству $L$. Поэтому мы будем считать, что $r$ и $t$ являются именно теми числами, существование которых утверждается доказываемой леммой.

Пусть теперь $f \in L_{0}$ удовлетворяет условиям леммы. Многочлен $f$ полилинейный, и существует некоторый многочлен

$$
g=\left[f_{0}, z_{0}\right]+\sum_{k=1}^{s}\left[f_{k}, x_{i_{k}}\right] \in P^{\prime \prime},
$$

получающийся из $f$ переименованием переменных. Нам достаточно доказать, что $g \in \Gamma^{\prime \prime}$.

При этом можно считать, что выполнено следующее условие. Пусть имеется некоторый многочлен

$$
g^{\prime}=\left[f_{0}^{\prime}, z_{0}\right]+\sum_{k=1}^{s^{\prime}}\left[f_{k}^{\prime}, x_{i_{k}^{\prime}}\right] \in P^{\prime \prime}
$$

и при этом $\max \left(\operatorname{vr}\left(g^{\prime}\right) \cap X\right)<\max (\operatorname{vr}(g) \cap X)$. Пусть также для любого разбиения $A_{1} \sqcup \cdots \sqcup A_{r} \sqcup B_{1} \sqcup \cdots \sqcup B_{t}=\operatorname{vr}\left(g^{\prime}\right)$ вьполняется

$$
S_{A_{1}} \ldots S_{A_{r}} \Lambda_{B_{1}} \ldots \Lambda_{B_{t}} g^{\prime} \in \Gamma^{\prime \prime}
$$

Тогда $g^{\prime} \in \Gamma^{\prime \prime}$.

Выполнение такого условия , очевидно, эквивалентно проведению индукции по величине $\max (\operatorname{vr}(g) \cap X)$.

Можно считать, что $f_{0} \in P(n, m)$ для некоторых $n$ и $m$. Действительно, пусть $\operatorname{vr}(g)=\left\{t_{1}, \ldots, t_{c}, t_{c+1}, \ldots, t_{c+d}, z_{0}\right\}$, где $t_{1}<\cdots<t_{c+d}, t_{1}, \ldots, t_{c} \in X$ и $t_{c+1}, \ldots, t_{c+d} \in Y$. Рассмотрим такой гомоморфизм $\varphi: F^{\prime \prime} \rightarrow F^{\prime \prime}$, что $\varphi\left(z_{0}\right)=z_{0}$, $\varphi\left(t_{k}\right)=x_{k}, k=1, \ldots, c$, и $\varphi\left(t_{c+k}\right)=y_{k}, k=1, \ldots, d$. Тогда в представлении, получающемся из представления (3) действием гомоморфизма $\varphi$, индукционный параметр $\max (\operatorname{vr}(\varphi(g)) \cap X)$ не превосходит исходного индукционного параметра $\max (\operatorname{vr}(g) \cap X)$. С другой стороны, условия $g \in \Gamma^{\prime \prime}$ и $\varphi(g) \in \Gamma^{\prime \prime}$ эквивалентны.

В силу формулы $K S_{n} \otimes K S_{m}=\bigoplus\left\{U_{D_{1}, D_{2}}:\left|D_{1}\right|=n,\left|D_{2}\right|=m\right\}$ для того чтобы показать, что $f_{0} \in A(\Gamma)$, достаточно доказать, что для любых двух таблищ $D_{1}$ и $D_{2}$, состоящих соответственно из $n$ и $m$ клеток, выполнено $U_{D_{1}, D_{2}} f_{0} \subset A(\Gamma)$.

Следуя схеме доказательства работы [1], рассмотрим два случая. 
СлучАй 1. В одну из таблиц $D_{1}$ или $D_{2}$ вкладьвается таблица $D_{t_{0}+1, r_{0}+1}$.

В этом случае размерность любого модуля $J_{C_{1}, C_{2}}$, где $D_{1}=\operatorname{tab}\left(C_{1}\right)$ и $D_{2}=$ $\operatorname{tab}\left(C_{2}\right)$, больше, чем $d^{2 m_{0}+1}$. Это означает, что при $n+m \leqslant 2 m_{0}+1$ для любого набора $\gamma$ такого, что $|\gamma|_{X}=n$ и $|\gamma|_{Y}=m$, выполняется $U_{D_{1}, D_{2}}(\gamma) \subset A(\Gamma)$.

Подслучай а). $D_{t_{0}+1, r_{0}+1}$ вкладывается в таблицу $D_{1}$.

Пусть

$$
\sum_{\sigma \in S_{m_{0}}} \alpha_{\sigma} x_{\sigma(1)} \ldots x_{\sigma\left(m_{0}\right)} \in U_{D_{t_{0}+1, r_{0}+1}}^{(X)}
$$

Тогда понятно, что

$$
\sum_{\sigma \in S_{m_{0}}} \alpha_{\sigma} y_{1} x_{\sigma(1)} y_{2} \ldots y_{m_{0}} x_{\sigma\left(m_{0}\right)} y_{m_{0}+1} \in\left(U_{D_{t_{0}+1, r_{0}+1}} \otimes K S_{m_{0}+1}\right)\left(\gamma^{\prime}\right)
$$

для $\gamma^{\prime}=(1,0, \ldots, 0,1)$, где $|\gamma|_{X}=m_{0}$ и $|\gamma|_{Y}=m_{0}+1$. Разлагая $K S_{m_{0}+1}$ на однородные компоненты $U_{D}$ и пользуясь замечанием в начале рассмотрения случая 1 , получаем $\left(U_{D_{t_{0}+1, r_{0}+1}} \otimes K S_{m_{0}+1}\right)\left(\gamma^{\prime}\right) \subset A(\Gamma)$ и

$$
\sum_{\sigma \in S_{m_{0}}} \alpha_{\sigma} y_{1} x_{\sigma(1)} y_{2} \ldots y_{m_{0}} x_{\sigma\left(m_{0}\right)} y_{m_{0}+1} \in A(\Gamma)
$$

Подслучай b). $D_{t_{0}+1, r_{0}+1}$ вкладывается в таблищу $D_{2}$.

Пусть

$$
\sum_{\sigma \in S_{m_{0}}} \alpha_{\sigma} y_{\sigma(1)} \ldots y_{\sigma\left(m_{0}\right)} \in U_{D_{t_{0}+1, r_{0}+1}}^{(Y)}
$$

Тогда по теореме ветвления (или по результатам работ [1] и [2]) имеем

$$
\sum_{\sigma \in S_{m_{0}}} \alpha_{\sigma} y_{m_{0}+1} y_{\sigma(1)} y_{2} \ldots y_{m_{0}} y_{\sigma\left(m_{0}\right)} y_{2 m_{0}+1} \in \underset{\substack{D_{t_{0}+1, r_{0}+1} \subset D \\|D|=2 m_{0}+1}}{ } U_{D}^{(Y)}
$$

Размерность любого неприводимого подмодуля модуля $U_{D}^{(Y)}$ из данной формулы больше, чем $d^{2 m_{0}+1}$. Поэтому $U_{D}^{(Y)} \subset A(\Gamma)$. Следовательно, мы имеем

$$
\sum_{\sigma \in S_{m_{0}}} \alpha_{\sigma} y_{m_{0}+1} y_{\sigma(1)} y_{2} \ldots y_{2 m_{0}} y_{\sigma\left(m_{0}\right)} y_{2 m_{0}+1} \in A(\Gamma)
$$

Так как $A(\Gamma)$ инвариантно относительно замен переменных из $Y$ на любые многочлены из $F^{\prime}$, то в силу формул (4) и (5) получаем, что $U_{D_{1}, D_{2}}(\gamma) \subset A(\Gamma)$ для любого $\gamma$ такого, что $|\gamma|_{X}=n$ и $|\gamma|_{Y}=m$. Отсюда имеем $U_{D_{1}, D_{2}} f_{0} \subset A(\Gamma)$. 
СлучАй 2. Ни в одну из таблищ $D_{1}$ или $D_{2}$ не вкладывается таблица $D_{t_{0}+1, r_{0}+1}$.

Аналогично тому, как это сделано в [1], легко показать, что в этом случае любой элемент из $U_{D_{1}, D_{2}} f_{0}$ представляется в виде линейной комбинации полиномов вида

$$
\left(\sigma_{1} \otimes \sigma_{2}\right) S_{A_{1}} \ldots S_{A_{2 r_{0}}} \Lambda_{B_{1}} \ldots \Lambda_{B_{2 t_{0}}} f_{0}
$$

где $\sigma_{1} \in S_{n}, \sigma_{2} \in S_{m}, A_{1} \sqcup \cdots \sqcup A_{r_{0}} \sqcup B_{1} \sqcup \cdots \sqcup B_{t_{0}}=\left\{x_{1}, \ldots, x_{n}\right\}$ и $A_{r_{0}+1} \sqcup \cdots$ $\cdots \sqcup A_{2 r_{0}} \sqcup B_{t_{0}+1} \sqcup \cdots \sqcup B_{2 t_{0}}=\left\{y_{1}, \ldots, y_{m}\right\}$.

Положим $A_{r}=\left\{z_{0}\right\}, V=S_{A_{1}} \ldots S_{A_{2 r_{0}}} \Lambda_{B_{1}} \ldots \Lambda_{B_{2 t_{0}}}$ и $V^{\prime}=S_{A_{1}} \ldots S_{A_{r}} \Lambda_{B_{1}} \ldots$ $\ldots \Lambda_{B_{t}}$.

Имеем по условию леммы

$$
V^{\prime} g=\left[V f_{0}, z_{0}\right]+\sum_{k=1}^{a}\left[g_{k}, x_{j_{k}}\right] \in \Gamma^{\prime \prime} \cap P^{\prime \prime} .
$$

Отсюда $V f_{0} \in A(\Gamma)$ и $U_{D_{1}, D_{2}} f_{0} \subset A(\Gamma)$.

Таким образом, оба случая рассмотрены и доказано, что $f_{0} \in A(\Gamma)$. Тогда по лемме 3

$$
h=\left[f_{0}, z_{0}\right]+\sum_{k=1}^{b}\left[h_{k}, x_{l_{k}}\right] \in \Gamma^{\prime \prime} \cap P^{\prime \prime} .
$$

Если $\operatorname{vr}(g) \cap X=\varnothing$, что соответствует случаю $n=0$, то $g=h \in \Gamma^{\prime \prime}$.

Пусть теперь $n \geqslant 1$. Согласно сделанному ранее индукционному предположению многочлен $\bar{g}$, получающийся из многочлена $g-h$ заменой $z_{0} \rightarrow y_{m+1}$, $x_{n} \rightarrow z_{0}$, лежит в $\Gamma^{\prime \prime}$. Отсюда $g-h \in \Gamma^{\prime \prime}$ и $g \in \Gamma^{\prime \prime}$. Лемма доказана.

\section{$\S 4 . T_{2}$-пространства}

1. Обозначения. Введем теперь обозначения, не зависимые от обозначений, введенных в $\S 2,3$, которые мы будем использовать до конща работы. Пусть $X$ и $Z$ - счетные непересекаюшиеся множества переменных, $X=\left\{x_{1}, \ldots, x_{n}, \ldots\right\}$, $Z=\left\{z_{1}, \ldots, z_{n}, \ldots\right\}, K$ - поле нулевой характеристики, $F=K\langle X \cup Z\rangle$ - алгебра без единицы и $L_{0}$ есть $T$-пространство алгебры $F$, порожденное коммутатором $\left[x_{1}, x_{2}\right]$. Аналогично, через $P$ обозначим множество полилинейных многочленов алгебры $F$, а через $T$ полугруппу эндоморфизмов алгебры $F$. Для любого однородного многочлена $f \in F$ через $\operatorname{deg}_{Z} f$ обозначим число $\sum_{i=1}^{\infty} \operatorname{deg}_{z_{i}} f$. Это число назовем $z$-степенью многочлена $f$. Через $F_{0}$ обозначим подпространство алгебры $F$, натянутое на слова четной $z$-степени, а через $F_{1}$ подпространство, натянутое на слова нечетной $z$-степени. Тогда $\left(F, F_{0}, F_{1}\right)$ есть $Z_{2}$-градуированная алгебра в смысле работы [3]. Через $M_{0}$ обозначим подпространство алгебры $F$, натянутое на буквы из $X$, а через $M_{1}$ подпространство, натянутое на буквы из $Z$.

Далее, следуя работе [3], введем следуюшие определения.

Через $T_{2}\left(S_{2}\right)$ обозначим полугруппу таких эндоморфизмов $\varphi$ алгебры $F$, что $\varphi\left(x_{i}\right) \in F_{0}$ и $\varphi\left(z_{j}\right) \in F_{1} \quad\left(\varphi\left(x_{i}\right) \in M_{0}\right.$ и $\left.\varphi\left(z_{j}\right) \in M_{1}\right)$ для любых $i$ и $j$.

Пространство $L$ назовем $T_{2}$-пространством $\left(S_{2}\right.$-пространством $)$, если из $f \in$ $L$ и $\varphi \in T_{2}\left(\varphi \in S_{2}\right)$ следует, что $\varphi(f) \in L$. 
2. Инволюция Кемера. Для двух подпространств $L_{1}$ и $L_{2}$ эквивалентность $L_{1} \simeq_{\Im} L_{2}$ считаем вьполненной, если наибольшие $\Im$-пространства, содержашиеся в $L_{1}$ и в $L_{2}$ соответственно, совпадают. Здесь знак $\Im$ обозначает один из символов $T$ или $T^{*}$.

Пусть $Z=\bigsqcup_{i=1}^{t} T_{i}$, где $T_{i}$ - счетные множества. Обозначим через $I_{r}$ идеал алгебры $F$, порожденный переменными $\left\{x_{r+1}, x_{r+2}, \ldots\right\}$, а через $J_{i}$ идеал алгебры $F$, порожденный многочленами $a_{1} f a_{2}+a_{2} f a_{1}$, где $a_{1}, a_{2} \in T_{i}$ и $f \in F \cup\{1\}$. Через $J$ обозначим идеал алгебры $F$, порожденный переменными из $Z$. Имеем $J_{i} \subset J$ для любого $i=1, \ldots, t$.

Имеет место

Лемма 6. Пусть $r \geqslant 3$. Тогда $L_{0}+I_{r}+J \simeq_{T} L_{0}$.

ДокАЗАТЕльство. Пусть $V$ - наибольшее $T$-пространство, содержашееся в $L_{0}+I_{r}+J$, и $f$ - полилинейный многочлен из $V$, зависящий от переменной $x_{1}$. Представим $f$ в виде $f=x_{1} g+f_{1}$, где $f_{1} \in L_{0}$ и $x_{1} \notin \operatorname{vr}(g)$. Пусть $\varphi: F \rightarrow$ $K\left\langle x_{1}, x_{2}, x_{3}\right\rangle \subset F$ - гомоморфизм такой, что $\varphi\left(x_{1}\right)=x_{1}$ и $\varphi\left(x_{i}\right) \in K\left\langle x_{2}, x_{3}\right\rangle$ при $i \geqslant 2$. Тогда $\varphi(f)=x_{1} \varphi(g)+\varphi\left(f_{1}\right) \in V \subset L_{0}+I_{r}+J$. Так как $\operatorname{vr}(\varphi(f)) \subset\left\{x_{1}, x_{2}, x_{3}\right\}$, то $\varphi(f) \in L_{0}$. Но $\varphi\left(f_{1}\right) \in L_{0}$, и поэтому $x_{1} \varphi(g) \in L_{0}$. Так как $\varphi(g)$ не зависит от $x_{1}$, то $\varphi(g)=0$. Вследствие произвольности $\varphi$, удовлетворяющего перечисленным вьше условиям, и того, что свободная двупорожденная алгебра не является РІ-алгеброй, получаем $g=0$. Отсюда $f=f_{1} \in L_{0}$. Когда полином $f \in V$ произвольный, нужно разложить его на однородные компоненты $f_{i}$, каждая из которых принадлежит $V$, а затем рассмотреть полиномы $f_{i}^{\prime}$, являюшиеся полными линеаризациями полиномов $f_{i}$. Тогда $f_{i}^{\prime} \in V$ и по доказанному $f_{i}^{\prime} \in L_{0}$. Отсюда в силу нулевой характеристики основного поля все $f_{i} \in L_{0}$ и $f \in L_{0}$. Следовательно, $V=L_{0}$. Лемма доказана.

Доказательство предыдущей леммы изложено так подробно потому, чтобы показать способ работы с отношениями $\simeq_{T}$ и $\simeq_{T^{*}}$, который будет применятся в этом параграфе.

Справедлива

ТЕОрема 4. Для любого ненулевого T-пространства $L \subset L_{0}$ существуют такие числа $r$ и $t$, что для любого $T$-пространства $\Gamma$, для которого $L \subset \Gamma \subset$ $L_{0}$, выполнена әквивалентность

$$
\Gamma+I_{r}+J_{t} \simeq_{T} \Gamma
$$

ДОКАЗАТЕЛЬСТВо. Выберем числа $r$ и $t$ в соответствии с леммой 5 так, чтобы $r \geqslant 3$. Пусть $V$ - наибольшее $T$-пространство, содержашееся в $\Gamma+I_{r}+J_{t}$. Имеем $\Gamma+I_{r}+J_{t} \subset L_{0}+I_{r}+J$. Тогда по лемме 6 получаем $V \subset L_{0}$. Теперь мы находимся в условиях леммы 5 и получаем, что $V=\Gamma$. Теорема доказана.

На множестве $P$ определим операцию sgn следующим образом. Пусть $u=$ $u_{0} z_{i_{\sigma(1)}} u_{1} \ldots u_{n-1} z_{i_{\sigma(n)}} u_{n} \in P$, где $u_{0}, \ldots, u_{n}$ - слова над алфавитом $X, i_{1}<\cdots$ $\cdots<i_{n}$ и $\sigma \in S_{n}$. Тогда положим $\operatorname{sgn}(u)=\operatorname{sgn}(\sigma)$ и продолжим $\operatorname{sgn}$ по линейности на все $P$. Для $f \in P$ положим также $f^{*}=\operatorname{sgn}(f) f$. Если $L$ есть $T_{2}$-пространство, 
то через $L^{*}$ обозначим $T_{2}$-пространство, порожденное как $S_{2}$-пространство множеством $(P \cap L)^{*}$. Пусть $\varphi$ - эндоморфизм алгебры $F$ такой, что для любых букв $x$ из $X$ и $z$ из $Z$ многочлен $\varphi(x)$ есть слово из $F_{0}$, а многочлен $\varphi(z)$ есть слово из $F_{1}$. Рассмотрим полилинейное слово $u=u_{0} z_{i_{\sigma(1)}} u_{1} \ldots u_{n-1} z_{i_{\sigma(n)}} u_{n}$, где $u_{0}, \ldots, u_{n}-$ слова над алфавитом $X, i_{1}<\cdots<i_{n}$ и $\sigma \in S_{n}$. Пусть $v$-слово, полученное некоторой перестановкой букв слова $u_{0} \ldots u_{n}$, такое, что номера индексов при $x$ в слове $v$ возрастают слева направо. Тогда имеем

$$
\begin{aligned}
\operatorname{sgn}(\varphi(u)) & =\operatorname{sgn}\left(\varphi\left(u_{0} \ldots u_{n} z_{i_{\sigma(1)}} \ldots z_{i_{\sigma(n)}}\right)\right)=\operatorname{sgn}(\sigma) \operatorname{sgn}\left(\varphi\left(v z_{i_{1}} \ldots z_{i_{n}}\right)\right) \\
& =\operatorname{sgn}\left(\varphi\left(v z_{i_{1}} \ldots z_{i_{n}}\right)\right) \operatorname{sgn}(u) .
\end{aligned}
$$

Домножая это равенство на $\varphi(u)$, получаем

$$
\begin{aligned}
\varphi(u)^{*} & =\operatorname{sgn}(\varphi(u)) \varphi(u)=\operatorname{sgn}\left(\varphi\left(v z_{i_{1}} \ldots z_{i_{n}}\right)\right) \operatorname{sgn}(u) \varphi(u) \\
& =\operatorname{sgn}\left(\varphi\left(v z_{i_{1}} \ldots z_{i_{n}}\right)\right) \varphi\left(u^{*}\right) .
\end{aligned}
$$

При этом моном $v z_{i_{1}} \ldots z_{i_{n}}$ зависит только от $\operatorname{vr}(u)$. Следовательно, верна

Лемма 7. Если L есть $T_{2}$-пространство, то $L^{*}$ тоже $T_{2}$-пространство.

Если в этой лемме заменить выражение " $T_{2}$-пространство" на выражение " $T_{2}$ идеал", то получится утверждение, доказанное в [3]. $T_{2}$-пространство $L$ назовем $T^{*}$-пространством, если $L=M^{*}$ для некоторого $T$-пространства $M$.

Пусть $I_{r, t}$ - идеал алгебры $F$, порожденньй переменньми

$$
\left\{x_{r+1}, \ldots\right\} \cup\left\{z_{t+1}, \ldots\right\} .
$$

Справедлива

ТЕОРема 5. Для любого ненулевого T-пространства $L \subset L_{0}$ существуют такие числа $r$ и $t$, что для любого T-пространства $\Gamma$, для которого $L \subset \Gamma \subset$ $L_{0}$, выполнено

$$
\Gamma^{*}+I_{r, t} \simeq_{T^{*}} \Gamma^{*}
$$

ДокАЗАТЕЛЬСТво. Выберем числа $r$ и $t$ в соответствии с теоремой 4 . Следуя работе [3], введем обозначения: $I_{X, r+1}^{-}-$идеал алгебры $F$, порожденный элементами

$$
\sum_{\sigma \in S_{r+1}} \operatorname{sgn} \sigma u_{0} x_{i_{\sigma(1)}} u_{1} \ldots u_{r} x_{i_{\sigma(r+1)}} u_{r+1}
$$

$I_{Z, t+1}^{-}-$идеал алгебры $F$, порожденньй элементами

$$
\sum_{\sigma \in S_{t+1}} \operatorname{sgn} \sigma v_{0} z_{j_{\sigma(1)}} v_{1} \ldots v_{r} z_{j_{\sigma(t+1)}} v_{t+1}
$$

где $u_{0}, \ldots, u_{r+1}, v_{0}, \ldots, v_{t+1} \in F \cup\{1\}$. Тогда $\Gamma^{*}+I_{X, r+1}^{-}+I_{Z, t+1}^{-} \simeq_{T^{*}} \Gamma^{*}$. Пусть $U$ - наибольшее $T^{*}$-пространство, содержашееся в $\Gamma^{*}+I_{r, t}$. Используя стандартные соображения о полиномах Капелли (см. [3]), получаем $U \subset \Gamma^{*}+$ $I_{X, r+1}^{-}+I_{Z, t+1}^{-}$. Так как $U$ является $T^{*}$-идеалом, то $U=\Gamma^{*}$. Теорема доказана. 
3. Редукция к локальному случаю. Пусть $u, v$-мономы алгебры $F$. Через $[u, v]_{2}$ обозначим полином $[u, v]$, если $\operatorname{deg}_{Z} u \cdot \operatorname{deg}_{Z} v$-четное число, или $u v+v u$, если $\operatorname{deg}_{Z} u \cdot \operatorname{deg}_{Z} v$-нечетное число. Для произвольных полиномов $f$ и $g$ выражение $[f, g]_{2}$ определим по линейности. Имеет место соотношение $([f, g])^{*}=\left[f^{*}, g^{*}\right]_{2}$, где $f, g \in P$ и $\operatorname{vr}(f) \cap \operatorname{vr}(g)=\varnothing$.

Для $T$-идеала $I$ алгебры $F$ через $[I, F]$ обозначим подпространство алгебры $F$, порожденное как линейное пространство элементами вида $[g, f]$, где $g \in I$ и $f \in$ $F$. Легко проверить, что $[I, F]$ есть $T$-пространство. Аналогично, для любого $T_{2}$-идеала $\Gamma$ алгебры $F$ через $[\Gamma, F]_{2}$ обозначим подпространство алгебры $F$, порожденное как линейное пространство элементами вида $[g, f]_{2}$, где $g \in \Gamma$ и $f \in F$. Легко проверить, что $[\Gamma, F]_{2}$ есть $T_{2}$-пространство. Действительно, имеем $[\Gamma, F]_{2}=\left(\left[\Gamma^{*}, F\right]\right)^{*}$. Здесь $\left[\Gamma^{*}, F\right]$ есть $T_{2}$-пространство, и, применяя операцию ${ }^{*}$, опять получаем $T_{2}$-пространство в силу леммы 7 . Имеем также $[I, F] \in L_{0}$ и $[\Gamma, F]_{2} \in L_{0}^{*}$.

Через $M_{n}$ обозначим $T$-идеал алгебры $F$, состоящий из тождеств алгебры матриц размера $n \times n$ над полем нулевой характеристики $K$.

Пусть $0 \neq L_{1} \subset \cdots \subset L_{i} \subset \cdots$-цепочка $T$-пространств. Если существует $i$ такое, что $L_{i} \backslash L_{0} \neq \varnothing$, то по теореме 1 получаем, что $L_{i}$ содержит ненулевой $T$-идеал. Возможен также случай, когда все $L_{i}$ содержатся в $L_{0}$. Мы будем доказывать стабилизацию только во втором случае, так как решение в первом случае получается некоторьм упрощением конструкции. Более формальный способ - свести все ко второму случаю, профильтровать по $L_{0}$ и воспользоваться леммой 2 и теоремой Кемера.

Проблему стабилизации цепочек $T$-пространств, каждое из которых содержится в $L_{0}$ (т. е. второй случай), назовем ограниченной проблемой ШІехта.

Следуя работе [3], докажем следуюший вариант редукции ограниченной проблемы Шпехта.

ТЕОРема 6. Предположим, что для любых иисел $n, r, t$ и для любой возрастающей чепочки $T_{2}$-пространств $\left[M_{n}, F\right]_{2} \subset V_{1} \subset \cdots \subset V_{i} \subset \cdots$ чепочка пространств $V_{1} \cap A \subset \cdots \subset V_{i} \cap A \subset \cdots$, где $A=K\left\langle x_{1}, \ldots, x_{r}, z_{1}, \ldots, z_{t}\right\rangle$, стабилизируется. Тогда ограниченная проблема Шпехта решается положительно.

ДоКАЗАТЕЛЬСТво. Предположим противное, и пусть $0 \neq L_{1} \subset \cdots \subset L_{i} \subset \cdots$ $\cdots \subset L_{0}$ - бесконечная строго возрастающая цепочка $T$-пространств. Так как $L_{1} \neq 0$, то по теореме 2 сушествует ненулевой $T$-идеал $I$ такой, что $[I, F] \subset L_{1}$. Тогда $\left[I^{*}, F\right]_{2} \subset L_{1}^{*}$. По теореме 5 и предложению 2 из работы [3] сушествуют натуральные числа $r$ и $t$ такие, что для любого $i \geqslant 1$ имеем $L_{i}^{*}+I_{r, t} \simeq_{T^{*}} L_{i}^{*}$ и $I^{*}+I_{r, t} \simeq_{T^{*}} I^{*}$.

Пусть $A=K\left\langle x_{1}, \ldots, x_{r}, z_{1}, \ldots, z_{t}\right\rangle$ - подалгебра алгебры $F$. Тогда $L_{i}^{*}+I_{t, r}=$ $L_{i}^{*} \cap A+I_{r, t}$ (в силу однородности) и цепочка пространств

$$
L_{1}^{*} \cap A \subset \cdots \subset L_{i}^{*} \cap A \subset \cdots
$$

строго возрастает. Действительно, если $L_{i}^{*} \cap A=L_{i+1}^{*} \cap A$, то $L_{i}^{*}+I_{r, t}=L_{i+1}^{*}+$ $I_{r, t}$. Отсюда имеем $L_{i+1}^{*} \subset L_{i}^{*}+I_{r, t}$ и, следовательно, $L_{i+1}^{*}=L_{i}^{*}$. Поэтому 
$L_{i+1}=L_{i}$. Противоречие. Пусть $V_{i}$ - наибольшее $T_{2}$-пространство, содержашееся в $\left(L_{i}^{*}+I_{r, t}\right) \cap L_{0}^{*}$. Тогда $V_{i} \cap A=L_{i}^{*} \cap A$ и цепочка $V_{1} \cap A \subset \cdots \subset V_{i} \cap A \subset \cdots$ строго возрастает. Пусть также $\Gamma$ - наибольший $T$-идеал, содержашийся в $I^{*}+I_{r, t}$. Как показано в лемме 7 работы [3], сушествует $n$ такое, что $M_{n} \subset \Gamma$. Для того чтобы прийти к противоречию, надо показать, что $\left[M_{n}, F\right]_{2} \subset V_{1}$. Так как $\left[M_{n}, F\right]_{2}$ есть $T_{2}$-пространство и $\left[M_{n}, F\right]_{2} \subset L_{0}^{*}$, то достаточно показать, что $\left[M_{n}, F\right]_{2} \subset L_{1}^{*}+I_{r, t}$.

Пусть $g \in M_{n} \cap A$, a $f \in A$. Так как $M_{n} \cap A \subset I^{*}$, то $g \in I^{*}$ и $[g, f]_{2} \in\left[I^{*}, F\right]_{2}$. Вспоминая, что $\left[I^{*}, F\right]_{2} \subset L_{1}^{*}$, получаем $\left[M_{n}, F\right]_{2} \subset L_{1}^{*}+I_{r, t}$ и $\left[M_{n}, F\right]_{2} \subset V_{1}$. Теорема доказана.

\section{§5. Квазимногочлены}

1. Определение квазимногочленов. Выделим в $T$ подполугруппу $T_{2}^{\prime}$ подстановок вида $x \rightarrow g x h$, где $x \in X \cup Z, \operatorname{a~deg}_{Z} g$ и $\operatorname{deg}_{Z} h$-четные числа. Очевидно, $T_{2}^{\prime} \subset T_{2}$. Подстановка $\alpha \in T$ называется однородной, если $\alpha(y)$ - однородньй многочлен для любого $y \in X \cup Z$.

Пусть $X_{r}=\left\{x_{k}^{(i, j)}: k \in \mathbb{N}, 1 \leqslant i, j \leqslant r\right\}$ и $Z_{r}=\left\{z_{k}^{(i, j)}: k \in \mathbb{N}, 1 \leqslant i, j \leqslant r\right\}-$ множества коммутативных переменных, а $K\left[X_{r} \cup Z_{r}\right]$ - свободная коммутативная алгебра с единицей. Обозначим через $\operatorname{GM}(r)$ подалгебру алгебры $K\left[X_{r} \cup Z_{r}\right]_{r}$, порожденную матрицами

$$
\bar{x}_{k}=\sum_{1 \leqslant i, j \leqslant r} x_{k}^{(i, j)} e_{i j}, \quad \bar{z}_{k}=\sum_{1 \leqslant i, j \leqslant r} z_{k}^{(i, j)} e_{i j}
$$

и единичной матрицей. Алгебра $\operatorname{GM}(r)$ естественным образом наделяется структурой $T$-пространства (см. [9]).

Пусть $\Theta=\left\{\sigma_{1}, \tau_{1}, \ldots, \sigma_{n}, \tau_{n}, \ldots\right\}$, и пусть $Q(r)=\mathrm{GM}(r) *_{K} K\langle\Theta\rangle$ - свободное произведение алгебр. Элементы этой алгебры называются квазимногочленами. Пусть $Q_{0}(r)$ - подпространство $Q(r)$, натянутое на квазимногочлены вида $f \theta$, где $f \in Q(r)$, а $\theta \in \Theta$. Для любого однородного квазимногочлена $f$ через $\operatorname{deg}_{\Theta} f$ обозначим количество вхождений букв из множества $\Theta$ в произвольный моном из $f$, если $f \neq 0$, а иначе положим $\operatorname{deg}_{\Theta} f=0$. Это число назовем $\theta$-степенью квазимногочлена $f$.

Для $n \in \mathbb{N}$ через $\Theta_{n}$ обозначим подпространство алгебры $Q(r)$, натянутое на однородные квазимногочлены $f$ такие, что $\operatorname{deg}_{\Theta} f \geqslant n$. Очевидно, каждое $\Theta_{n}-$ идеал алгебры $Q(r)$ и $\Theta_{0}=Q(r)$.

Далее будем считать, что $x_{i}=\bar{x}_{i}+\sigma_{i}$ и $z_{j}=\bar{z}_{j}+\tau_{j}$. Такие равенства не противоречат свободе переменных $x_{i}, z_{j}, \sigma_{i}, \tau_{j}$ и индуцируют вложение $F \subset Q(r)$. Отображение $y: \Theta \rightarrow X \cup Z$ зададим формулами $y\left(\sigma_{i}\right)=x_{i}$ и $y\left(\tau_{j}\right)=z_{j}$, и пусть $\theta=y^{-1}$. Пусть $\lambda: F \rightarrow \operatorname{GM}(r)$ - гомоморфизм, определенный формулами $\lambda\left(x_{i}\right)=\bar{x}_{i}$ и $\lambda\left(z_{i}\right)=\bar{z}_{i}$.

Мы считаем, что переменные $x_{k}^{(i, j)}, \sigma_{k}$ соответствуют переменной $x_{k}$, а переменные $z_{k}^{(i, j)}, \tau_{k}$ переменной $z_{k}$. Для любого однородного элемента $f$ любой из алгебр $\operatorname{GM}(r)$ или $Q(r)$ его $z$-степенью $\left(\operatorname{deg}_{Z} f\right)$ назовем суммарную степень $f$ по всем переменным, соответствуюшим переменным из $Z$. 
2. Действия полугрупп $T$ и $T_{2}$. Если $\alpha \in T$ - подстановка типа $y \rightarrow g_{y}$, где $y \in X \cup Z$ и $g_{y} \in F$, то положим $\lambda(y)^{\alpha}=\lambda\left(g_{y}\right)$ и $\theta(y)^{\alpha}=g_{y}-\lambda\left(g_{y}\right)$. Это действие преврашает $Q(r)$ и все его идеалы $\Theta_{n}$ в $T$-пространства. При этом таким образом определенное действие на $F$ совпадает с обычным действием $T$ на $F$.

Определим теперь действие на $Q_{0}(r)$, отличное от только что введенного. Пусть $g \theta$ - элемент из $Q_{0}(r)$, где $\theta \in \Theta, g$ - однородный квазимногочлен и $\alpha \in T_{2}-$ подстановка. Положим $\beta=(-1)^{\operatorname{deg}_{Z} g}$. Предположим сначала, что $\alpha(y(\theta))=$ $y_{1} \ldots y_{k}$, где $y_{i} \in X \cup Z$. Для $j=1, \ldots, k$ положим $n_{j}=\operatorname{deg}_{Z} y_{1} \ldots y_{j}$ и $m_{j}=$ $\operatorname{deg}_{Z} y_{j+1} \ldots y_{k}$. Тогда

$$
(g \theta)^{(\alpha)}=\sum_{j=1}^{k} \beta^{m_{j}}(-1)^{m_{j} n_{j}} y_{j+1} \ldots y_{k} g^{\alpha} y_{1} \ldots y_{j-1} \theta\left(y_{j}\right) .
$$

Теперь пусть $\alpha(y(\theta))=\gamma_{1} y_{1}+\cdots+\gamma_{k} y_{k}$, где $\gamma_{i} \in K$. Тогда

$$
(g \theta)^{(\alpha)}=\sum_{j=1}^{k} g^{\alpha}\left(\gamma_{1} \theta\left(y_{1}\right)+\cdots+\gamma_{k} \theta\left(y_{k}\right)\right) .
$$

Действие всего $T_{2}$ на $Q_{0}(r)$ определим, продолжая приведенные выше частные случаи так, чтобы $Q_{0}(r)$ было $K T_{2}$-модулем.

Подпространство пространства $Q_{0}(r)$, замкнутое относительного такого действия для любого $\alpha \in T_{2}$, назовем ограниченным $T_{2}$-пространством.

Введем следующее определение. Пусть имеется элемент из $L_{0}^{*}$

$$
f=\sum_{i=1}^{k}\left[g_{i}, y_{i}\right]_{2}
$$

где $g_{i} \in F$, а $y_{i} \in X \cup Z$ и $f_{0}=\sum_{i=1}^{k} g_{i} \theta\left(y_{i}\right)$. Тогда считаем, что $f \rightarrow_{R} f_{0}$. Квазимногочлен $f_{0}$ строится однозначно по представлению (7). Вообще говоря, могут сушествовать различные $f_{1}, f_{2}$, для которых $f \rightarrow_{R} f_{1}$ и $f \rightarrow_{R} f_{2}$. Однако если $f_{1} \rightarrow_{R} f_{0}$ и $f_{2} \rightarrow_{R} f_{0}$, то $f_{1}=f_{2}$.

Важную связь между $F$ и $Q_{0}(r)$ устанавливает

ЛЕмма 8. Пусть $f \in F, f \rightarrow_{R} f_{0} u f_{0}=\sum_{i=1}^{l} g_{i} \theta_{i}$, əде $g_{i} \in Q(r), \theta_{i} \in \Theta u$ $i_{1} \neq i_{2} \Longrightarrow \theta_{i_{1}} \neq \theta_{i_{2}}$. Тогда все $g_{i}$ принадлежсат $F$.

ДоказАтЕльство. Имеет место представление $f=\sum_{i=1}^{k}\left[g_{i}^{\prime}, y_{i}^{\prime}\right]$, где $g_{i}^{\prime} \in F$, $y_{i}^{\prime} \in X \cup Z$ и $f_{0}=\sum_{i=1}^{k} g_{i}^{\prime} \theta\left(y_{i}^{\prime}\right)$. Группируя элементы $g_{i}^{\prime} \theta\left(y_{i}^{\prime}\right)$ по последней справа переменной, получаем, что каждое $g_{i}$ есть сумма некоторых $g_{j}^{\prime}$, т. е. принадлежит $F$. Лемма доказана.

Имеет место

ЛЕмма 9. Пусть $f \rightarrow_{R} f_{0}, \quad \alpha \in T_{2} u f_{0}^{(\alpha)}=\sum_{i=1}^{k} h_{i} \theta_{i}$, әде $h_{i} \in Q(r)$, $\theta_{i} \in \Theta u i_{1} \neq i_{2} \Longrightarrow \theta_{i_{1}} \neq \theta_{i_{2}}$. Тогдa $f^{\alpha}=\sum_{i=1}^{k}\left[h_{i}, y\left(\theta_{i}\right)\right]_{2}$ u $h_{i} \in F$. При ${ }_{\ni}$ том $f^{\alpha} \rightarrow_{R} f_{0}^{(\alpha)}$. 
ДокАЗАТЕЛЬСТво. Пусть $f_{0}=\sum_{j=1}^{l} g_{j} \theta_{j}^{\prime}$, где $g_{j} \in F, \theta_{j}^{\prime} \in \Theta$, и $f=$ $\sum_{j=1}^{l}\left[g_{j}, y\left(\theta_{j}^{\prime}\right)\right]_{2}$. Легко проверить, что если $g \in F-$ однородный полином и $u=y_{1} \ldots y_{k}-$ моном, где $y_{i} \in X \cup Z$, то справедлива формула

$$
[g, u]_{2}=\sum_{j=1}^{k} \beta^{m_{j}}(-1)^{m_{j} n_{j}}\left[y_{j+1} \ldots y_{k} g y_{1} \ldots y_{j-1}, y_{j}\right]_{2}
$$

где $m_{j}=\operatorname{deg}_{Z} y_{j+1} \ldots y_{k}, n_{j}=\operatorname{deg}_{Z} y_{1} \ldots y_{j}$ и $\beta=(-1)^{\operatorname{deg}_{Z} g}$.

Выполним теперь подстановку ${ }^{\alpha}$ в многочлене $f$ и воспользуемся формулой (8) для преобразования коммутаторов. После этого сгруппируем члены $[\cdot, \cdot]_{2}$ по второму аргументу. Переходя от $f^{\alpha}$, представленном в таком виде, к $f^{\prime}$ такому, что $f^{\alpha} \rightarrow_{R} f^{\prime}$, получаем $f^{\prime}=f_{0}^{(\alpha)}=\sum_{i=1}^{k} h_{i} \theta_{i}$. Отсюда ясно, что $h_{i} \in F$ (лемма 8) и $f^{\alpha}=\sum_{i=1}^{k}\left[h_{i}, y\left(\theta_{i}\right)\right]_{2}$. Лемма доказана.

Эта лемма показывает, что действия ${ }^{\alpha}{ }_{\text {и }}{ }^{(\alpha)}$ в некотором смысле согласованы.

Пусть теперь $L \subset L_{0}^{*}$ - некоторое $T_{2}$-пространство. Положим $R(L)=\{g \in$ $Q_{0}(r)$ : существует $f \in L$ такое, что $\left.f \rightarrow_{R} g\right\}$. По лемме 9 получаем, что $R(L)$ есть ограниченное $T_{2}$-пространство.

Лемма 10. Пусть $L_{1}, L_{2} \subset L_{0}^{*}$ суть $T_{2}$-пространства и $R\left(L_{1}\right) \subset R\left(L_{2}\right)$. Тогда $L_{1} \subset L_{2}$.

ДокаЗАтЕльство. Пусть $f \in L_{1}$. Тогда $f \rightarrow_{R} f_{0}$ для некоторого $f_{0}$. По определению $R\left(L_{1}\right)$ имеем $f_{0} \in R\left(L_{1}\right)$. Но тогда $f_{0} \in R\left(L_{2}\right)$ и существует $g \in L_{2}$ такое, что $g \rightarrow_{R} f_{0}$. Сравнивая $f \rightarrow_{R} f_{0}$ и $g \rightarrow_{R} f_{0}$, получаем, что $f=g \in L_{2}$. Лемма доказана.

А.В. Гришин в работе [8] доказал следуюший результат.

Лемма 11. $F \cap \Theta_{n}=M_{r}^{n}$.

Из этой леммы и леммы 8 получаем, что справедлива

Лemma 12. $R\left(L_{0}^{*}\right) \cap \Theta_{2}=R\left(\left[M_{r}, F\right]_{2}\right)$.

ДокАЗАТЕльСТво. Пусть $f \rightarrow R f_{0}$ и $f_{0}=\sum_{i=1}^{k} g_{i} \theta_{i} \in \Theta_{2}$, где $i_{1} \neq i_{2} \Longrightarrow$ $\theta_{i_{1}} \neq \theta_{i_{2}}$. По лемме 8 получаем, что все $g_{i}$ принадлежат $F \cap \Theta_{1}=M_{r}$. Следовательно, $f=\sum_{i=1}^{k}\left[g_{i}, y\left(\theta_{i}\right)\right]_{2} \in\left[M_{r}, F\right]_{2}$ и $f_{0} \in R\left(\left[M_{r}, F\right]_{2}\right)$. Наоборот, если $f=\sum_{i=1}^{k}\left[g_{i}, y_{i}\right]_{2}$ и $f_{0}=\sum_{i=1}^{k} g_{i} \theta\left(y_{i}\right)$, где $g_{i} \in M_{r}$ и $y_{i} \in X \cup Z$, то $g_{i} \in F \cap \Theta_{1}$. Отсюда $f_{0} \in R\left(L_{0}^{*}\right) \cap \Theta_{2}$. Лемма доказана.

3. Разделение переменных по позициям. В дальнейшем удобно считать, что элементы некоторой алгебры следов коммутируют не только с общими матрицами, но и с элементами из $\Theta$. Согласно [9] сделаем это следующим образом. Введем множества переменных $X_{r, s}=\left\{x_{k, s}^{(i, j)}: k \in \mathbb{N}, 1 \leqslant i, j \leqslant r\right\}$ и $Z_{r, s}=\left\{z_{k, s}^{(i, j)}: k \in \mathbb{N}, 1 \leqslant i, j \leqslant r\right\}$. Положим

$$
x_{k}^{(s)}=\sum_{1 \leqslant i, j \leqslant r} x_{k, s}^{(i, j)} e_{i j}, \quad z_{k}^{(s)}=\sum_{1 \leqslant i, j \leqslant r} z_{k, s}^{(i, j)} e_{i j}
$$


Обозначим через $\operatorname{GM}(r, s)$ подалгебру алгебры $K\left[X_{r, s} \cup Z_{r, s}\right]_{r}$, порожденную элементами $x_{k}^{(s)}, z_{k}^{(s)}$ и единичной матрицей. Через $\operatorname{Tr}(r, s)$ обозначим подалгебру алгебры $K\left[X_{r, s} \cup Z_{r, s}\right]$, порожденную элементами $\operatorname{tr} u$ и $\operatorname{tr} v \operatorname{tr} w$, где $u, v, w \in$ $\operatorname{GM}(r, s)$ и $\operatorname{deg}_{Z} u, \operatorname{deg}_{Z} v+\operatorname{deg}_{Z} w-$ четные числа. Наконец, пусть $\operatorname{Tr} \operatorname{GM}(r, s)-$ подалгебра алгебры $K\left[X_{r, s} \cup Z_{r, s}\right]_{r}$, порожденная алгебрами $\operatorname{GM}(r, s)$ и $\operatorname{Tr}(r, s)$. Пусть $\lambda_{i}: F \rightarrow \operatorname{GM}\left(r_{i}, i\right)$ - гомоморфизм, определенный формулами $\lambda_{i}\left(x_{k}\right)=x_{k}^{(i)}$ и $\lambda_{i}\left(z_{k}\right)=z_{k}^{(i)}$.

Считаем, что переменные $x_{k, s}^{(i, j)}, \sigma_{k}$ соответствуют переменной $x_{k}$, а переменные $z_{k, s}^{(i, j)}, \tau_{k}$ соответствуют переменной $z_{k}$. Степенью некоторого однородного квазимногочлена или многочлена $f$ относительно переменной $y \in X \cup Z$ (множества переменных $H$ ) назовем его суммарную степень по всем переменным, соответствуюшим переменной $y$ (переменным из $H$ ), и обозначим это число через $\operatorname{deg}_{y} f$ $\left(\operatorname{deg}_{H} f\right)$.

Для $\alpha \in T_{2}$ и $f \in \operatorname{Tr} \operatorname{GM}(r, s)$ или $f \in \operatorname{Tr}(r, s)$ через $f^{\alpha}$ обозначим естественное действие $\alpha$, когда переменной $x_{k}$ соответствует матрица $x_{k}^{(s)}$, а переменной $z_{k}-$ матрица $z_{k}^{(s)}$ (см. [9]).

Пусть $r_{1}, \ldots, r_{n}$ - натуральные числа. Через $R\left\langle r_{1}, \ldots, r_{n}\right\rangle$ обозначим линейное пространство, натянутое на элементы вида $f_{1} \theta_{1} \ldots f_{n} \theta_{n}$, где $f_{i} \in \operatorname{Tr} \operatorname{GM}\left(r_{i}, i\right)$ и $\theta_{i} \in \Theta$. Элементы этого пространства также назовем квазимногочленами. Через $\operatorname{Tr}\left\langle r_{1}, \ldots, r_{n}\right\rangle$ обозначим подалгебру алгебры $K\left[\bigcup_{i=1}^{n}\left(X_{r_{i}, i} \cup Z_{r_{i}, i}\right)\right]$, порожденную алгебрами $\operatorname{Tr}\left(r_{i}, i\right), i=1, \ldots, n$. Полугруппа $T_{2}$ стандартным образом действует на $\operatorname{Tr}\left\langle r_{1}, \ldots, r_{n}\right\rangle$.

Если $a=a_{1} \ldots a_{n} \in \operatorname{Tr}\left\langle r_{1}, \ldots, r_{n}\right\rangle$ и $f=f_{1} \theta_{1} \ldots f_{n} \theta_{n} \in R\left\langle r_{1}, \ldots, r_{n}\right\rangle$, где $a_{i} \in \operatorname{Tr}\left(r_{i}, i\right), f_{i} \in \operatorname{Tr} \operatorname{GM}\left(r_{i}, i\right)$ и $\theta_{i} \in \Theta$, то положим $a \cdot f=a_{1} f_{1} \theta_{1} \ldots a_{n} f_{n} \theta_{n}$. Как показал А. В. Гришин в работе [9], продолжая это действие по линейности, мы получим, что $R\left\langle r_{1}, \ldots, r_{n}\right\rangle$ есть унитарный $\operatorname{Tr}\left\langle r_{1}, \ldots, r_{n}\right\rangle$-модуль.

Теперь определим действие полугрупшы $T_{2}$ на $R\left\langle r_{1}, \ldots, r_{n}\right\rangle$, аналогичное действию $T_{2}$ на $Q_{0}(r)$. Однако определим это действие, используя некоторое обобщение пространства $R\left\langle r_{1}, \ldots, r_{n}\right\rangle$. Предположим, что мы имеем алгебры $A_{1}, \ldots, A_{n}$ и гомоморфизмы $\varphi_{i}: F \rightarrow A_{i}, i=1, \ldots, n$. Предположим дополнительно, что все алгебры $A_{1}, \ldots, A_{n}$ являются левыми $K T_{2}$-модулями и что все $\varphi_{i}$ есть $K T_{2}$-гомоморфизмы. Результат действия эндоморфизма $\alpha \in T_{2}$ на элемент $a_{i} \in A_{i}$ мы обозначим через $a^{\alpha}$. Мы считаем, что все алгебры $A_{i}$ являются $Z_{2}$-градуированныпи, т. е. $A_{i}=A_{i}^{0} \oplus A_{i}^{1}$, где $A_{i}^{0}$ и $A_{i}^{1}$ есть $T_{2}$-инвариантные подпространства такие, что $A_{i}^{0} A_{i}^{0} \subset A_{i}^{0}, A_{i}^{0} A_{i}^{1} \subset A_{i}^{1}, A_{i}^{1} A_{i}^{0} \subset A_{i}^{1}, A_{i}^{1} A_{i}^{1} \subset A_{i}^{1}, \varphi_{i}\left(F_{0}\right) \subset A_{i}^{0}$ и $\varphi_{i}\left(F_{1}\right) \subset A_{i}^{1}$.

Примерами таких отображений и алгебр служат отображения $\lambda_{i}$ и алгебры $\operatorname{Tr} \operatorname{GM}\left(r_{i}, i\right)$. Определим $R\left\langle A_{1}, \varphi_{1}, \ldots, A_{n}, \varphi_{n}\right\rangle$ как линейное пространство, натянутое на элементы вида $a=a_{1} \theta_{1} \ldots a_{n} \theta_{n}$, где $a_{i} \in A_{i}$ и $\theta_{i} \in \Theta$. Элемент $a$ назовем однороднылм, если каждое $a_{i}$ принадлежит либо $A_{i}^{0}$, либо $A_{i}^{1}$. Для однородного $a$ определим $\beta(a)=1$, если $a$ - четный элемент, и $\beta(a)=-1$, если $a$ нечетный.

Действие $K T_{2}$ на $R\left\langle A_{1}, \varphi_{1}, \ldots, A_{n}, \varphi_{n}\right\rangle$ определим следующим образом.

Для монома $u=y_{1} \ldots y_{l}$ алгебры $F$, где $y_{j} \in X \cup Z$, и для $i=1, \ldots, n-1$ 
положим

$$
\theta^{(i)}(u)=\sum_{j=1}^{l} \varphi_{i}\left(y_{1}\right) \ldots \varphi_{i}\left(y_{j-1}\right) \theta\left(y_{j}\right) \varphi_{i+1}\left(y_{j+1}\right) \ldots \varphi_{i+1}\left(y_{l}\right)
$$

Для произвольного $f \in F$ выражение $\theta^{(i)}(f)$ определим из соображений линейности.

Пусть $\alpha \in T_{2}$ - подстановка типа $y \rightarrow g_{y}, y \in X \cup Z$ и $a=a_{1} \theta_{1} \ldots a_{n} \theta_{n}$ однородный элемент, где $a_{i} \in A_{i}$ и $\theta_{i} \in \Theta$. Предположим сначала, что $\alpha\left(y\left(\theta_{n}\right)\right)=$ $y_{1} \ldots y_{l}$, где $y_{i} \in X \cup Z$.

Тогда положим

$$
\begin{aligned}
a^{(\alpha)}= & \sum_{j=1}^{l} \beta(a)^{m_{j}}(-1)^{m_{j} n_{j}} \varphi_{1}\left(y_{j+1} \ldots y_{l}\right)\left(\prod_{i=1}^{n-1} a_{i}^{\alpha} \theta^{(i)}\left(\alpha\left(y\left(\theta_{i}\right)\right)\right)\right) \\
& \times \varphi_{n}\left(y_{1} \ldots y_{j-1}\right) \theta\left(y_{j}\right),
\end{aligned}
$$

где $m_{j}=\operatorname{deg}_{Z} y_{j+1} \ldots y_{l}$ и $n_{j}=\operatorname{deg}_{Z} y_{1} \ldots y_{j}$.

Теперь пусть $\alpha(y(\theta))=\gamma_{1} y_{1}+\cdots+\gamma_{l} y_{l}$, где $\gamma_{i} \in K$ и $y_{i} \in X \cup Z$. Тогда

$$
a^{(\alpha)}=\prod_{i=1}^{n} a_{i}^{\alpha} \theta\left(\alpha\left(y\left(\theta_{i}\right)\right)\right)
$$

Действие $T_{2}$ на $R\left\langle A_{1}, \varphi_{1}, \ldots, A_{n}, \varphi_{n}\right\rangle$ определим, продолжая приведенные выше частные случаи, так, чтобы $R\left\langle A_{1}, \varphi_{1}, \ldots, A_{n}, \varphi_{n}\right\rangle$ было $K T_{2}$-модулем.

Любое подпространство $L$ пространства $R\left\langle A_{1}, \varphi_{1}, \ldots, A_{n}, \varphi_{n}\right\rangle$, замкнутое относительно такого действия, как и в случая подпространств пространства $Q_{0}(r)$, будем называть ограниченным $T_{2}$-пространством. Пусть $H \subset X \cup Z$. Если при этом $L$ порождено как ограниченное $T_{2}$-пространство элементами, являюшимися линейными комбинациями элементов вида $a_{1} \theta_{1} \ldots a_{n} \theta_{n}$, где $y\left(\theta_{i}\right) \in H$ и $a_{i} \in$ $\varphi(K\langle H\rangle)$, то $L$ называется ограниченным $H$-зависящим $T_{2}$-пространством.

Предположим, что у нас теперь имеются алгебры $B_{1}, \ldots, B_{n}$ и гомоморфизмы $\psi_{1}, \ldots, \psi_{n}$, аналогичные алгебрам $A_{1}, \ldots, A_{n}$ и гомоморфизмам $\varphi_{1}, \ldots, \varphi_{n}$.

Пусть $\pi_{i}: A_{i} \rightarrow B_{i}$ - такие гомоморфизмы, являющиеся $K T_{2}$-гомоморфизмами, что диаграммы

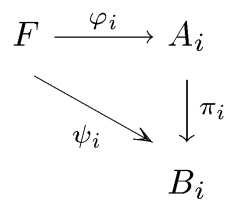

коммутативны.

Рассмотрим отображение $\pi: R\left\langle A_{1}, \varphi_{1}, \ldots, A_{n}, \varphi_{n}\right\rangle \rightarrow R\left\langle B_{1}, \psi_{1}, \ldots, B_{n}, \psi_{n}\right\rangle$, заданное формулой $\pi\left(a_{1} \theta_{1} \ldots a_{n} \theta_{n}\right)=\pi_{1}\left(a_{1}\right) \theta_{1} \ldots \pi_{n}\left(a_{n}\right) \theta_{n}$, где $a_{i} \in A_{i}$ и $\theta_{i} \in \Theta$. 
УТВЕРЖДЕНИЕ 1. Отображение $\pi$ является $K T_{2}$-гомоморфизмом.

Любое подпространство пространства $R\left\langle r_{1}, \ldots, r_{n}\right\rangle$, замкнутое относительного такого действия, назовем, как и случае пространства $Q_{0}(r)$, ограниченнылм $T_{2}$-пространством.

Пусть $c$ и $d$ - натуральные числа. Положим $G=\left\{x_{1}, \ldots, x_{c}, z_{1}, \ldots, z_{d}\right\}$. Пусть $H \subset X$. Через $R(H), \operatorname{Tr}(H), \operatorname{Tr} \mathrm{GM}_{i}(H)$ и $\mathrm{GM}_{i}(H)$ обозначим подпространства пространств $R\left\langle r_{1}, \ldots, r_{n}\right\rangle, \operatorname{Tr}\left\langle r_{1}, \ldots, r_{n}\right\rangle, \operatorname{Tr} \operatorname{GM}\left(r_{i}, i\right)$ и $\operatorname{GM}\left(r_{i}, i\right)$ соответственно, состоящие из квазимногочленов (многочленов), зависяших только от переменных, соответствуюших переменным из $G \cup H$ и имеющих относительно каждой переменной из $H$ степень, не большую единицы.

Пользуясь теоремой 6 , получаем, что для решения ограниченной проблемы Шпехта достаточно для любой цепочки ограниченных $T_{2}$-пространств $L_{1} \subset \ldots$ $\cdots \subset L_{i} \subset \cdots \subset R\left\langle r_{1}, \ldots, r_{n}\right\rangle$ доказать, что стабилизируется цепочка $L_{1} \cap$ $R(\varnothing) \subset \cdots \subset L_{i} \cap R(\varnothing) \subset \cdots$ для любого конечного $G$. При этом надо учесть лемму 10 и лемму 12 , замечая, что $Q_{0}(r) / \Theta_{2}$ изоморфно вкладывается в $R\langle r\rangle$ как $K T_{2}$-модуль.

\section{§6. Старшие части квазимногочленов}

В этом параграфе мы используем метод ширины, введенный А. В. Гришиным в работе [8].

1. Элементарные подпространства. Через $F^{e}$ обозначим алгебру, получающуюся из $F$ внешним присоединением единицы, а через $\bar{F}$ подалгебру алгебры $F^{e}$, натянутую как линейное пространство на мономы, зависящие только от переменных из $G$ и имеюшие четную $z$-степень. При этом продолжим гомоморфизмы $\gamma_{j}$ на все $\bar{F}$, полагая $\gamma_{j}\left(1_{\bar{F}}\right)$ равной единичной матрище размера $r_{j} \times r_{j}$.

Пусть $t \in X \cup Z$ - некоторая переменная и $g \in F$. Тогда $\alpha(t, g) \in T$ - такой эндоморфизм, что $\alpha(t, g)(y)=y$ при $y \in(X \cup Z) \backslash\{t\}$ и $\alpha(t, g)(t)=t g$. Заметим, что если $\operatorname{deg}_{Z} g$ - четное число, то $\alpha \in T_{2}^{\prime}$.

Фиксируем некоторое бесконечное множество переменных $S \subset X$ такое, что $S \cap G=\varnothing$.

Через $a=\left(a_{1}, \ldots, a_{k}\right)$ будем обозначать упорядоченные наборы. Величину $k$ будем называть длиной набора $a$ и обозначать через $|a|$. Через $\bar{a}$ обозначим неупорядоченное множество элементов, из которых состоит набор $a$. Для $i=1, \ldots, n$ через $i^{+}$обозначим число от 1 до $n$, равное $i+1$ по модулю $n$. Расстановкой числа $n$ назовем множество $\left\{j_{1}, \ldots, j_{q}\right\}$ такое, что $1 \leqslant j_{1}<\cdots<j_{q} \leqslant n$.

Пусть теперь $\eta=\left(t_{1}, \ldots, t_{q}\right)$ - набор различных переменных из $S, \sigma=\left\{j_{1}, \ldots\right.$ $\left.\ldots, j_{q}\right\}$ - расстановка числа $n$, где $j_{1}<\cdots<j_{q}$, и $\xi, \mu$-некоторые подмножества $S$ такие, что $\xi \cup \bar{\eta} \subset \mu, \xi \cap \bar{\eta}=\varnothing$ и $|\xi| \leqslant n$. Тогда через $R^{(\sigma, \eta, \xi, \mu)}$ обозначим подпространство пространства $R(\mu)$, натянутое на такие квазимногочлены $f=$ $f_{1} \theta_{1} \ldots f_{n} \theta_{n}$, где $f_{i} \in \operatorname{Tr} \operatorname{GM}\left(r_{i}, i\right), \theta_{i} \in \Theta$, что выполнены условия:

1 ) квазимногочлен $f$ имеет по каждой переменной из $\mu$ степень 1 ;

2 ) для $i=1, \ldots, q$ многочлен $f_{j_{i}}$ зависит от $t_{i}$;

$3)$ выполнена эквивалентность $(t \in \xi) \Longleftrightarrow\left(\theta(t)=\theta_{j}\right.$ для некоторого $j=$ $1, \ldots, n$ и $t \in S)$. 
Пусть $\sigma_{0}=\varnothing, \eta_{0}=\varnothing, \xi_{0}=\varnothing, \mu_{0}=\varnothing-$ пустые наборы и множества. Тогда $R^{\left(\sigma_{0}, \eta_{0}, \xi_{0}, \mu_{0}\right)}=R(\varnothing)$.

Следуя работе [8], позиции в квазимногочлене $f$ с номерами $j_{1}, \ldots, j_{q}$ назовем активныцми, а остальные пассивными относительно расстановки $\sigma$.

Пусть $j=1, \ldots, n$. Положим $\sigma_{j}=\left(j_{1}, \ldots, j_{m-1}, j, j_{m}, \ldots, j_{q}\right)$ и $m(\sigma, j)=m$, если $j_{m-1}<j<j_{m}$ для некоторого $m$, и $\sigma_{j}=\sigma$ и $m(\sigma, j)=0$, если такого $m$ не существует.

2. Операторы дифференцирования и умножения. Пусть теперь имеются попарно различные переменные $t_{1}, \ldots, t_{k} \in S \backslash \mu$. Определим оператор дифференцирования $D_{t_{1}, \ldots, t_{k}}^{\sigma}$.

Пусть $f=f_{1} \theta_{1} \ldots f_{n} \theta_{n} \in R^{(\sigma, \eta, \xi, \mu)}$ - однородный квазимногочлен, где $f_{i} \in$ $\operatorname{Tr} \operatorname{GM}\left(r_{i}, i\right), \theta_{i} \in \Theta$. Пусть $\alpha$ - подстановка типа $y \rightarrow y\left(1+t_{1}+\cdots+t_{k}\right), y \in G$. Через $h$ обозначим квазимногочлен, полученньй из $f$ аналогично квазимногочлену $f^{(\alpha)}$ по формулам (9)-(11), с тем лишь отличием, что операция ${ }^{\alpha}$ применяется только к многочленам $f_{i}$, стояшим на пассивных позищиях относительно расстановки $\sigma$. Тогда пусть $D_{t_{1}, \ldots, t_{k}}^{\sigma} f$ обозначает компоненту квазимногочлена $h$, линейную по переменным $t_{1}, \ldots, t_{k}$. Для произвольного $f \in R^{(\sigma, \eta, \xi, \mu)}$ квазимногочлен $D_{t_{1}, \ldots, t_{k}}^{\sigma} f$ определим по линейности.

Пусть $t \in \mu$ и имеется однородный многочлен $g \in \bar{F}$. При $\operatorname{deg} g \geqslant 1$ будем считать, что $g=\sum_{l=1}^{s} \gamma_{l} y_{1, l} \ldots y_{k, l} \in \bar{F}$, где $\gamma_{l} \in K$ и $y_{i, l} \in G$. При $\operatorname{deg} g \leqslant 0$ считаем, что $g=\gamma \cdot 1_{\bar{F}}$, где $\gamma \in K$.

Определим тогда оператор умножения $C_{t, g}^{\sigma}$, действуюший на $R^{(\sigma, \eta, \xi, \mu)}$ следующим образом. Пусть $f=f_{1} \theta_{1} \ldots f_{n} \theta_{n} \in R^{(\sigma, \eta, \xi, \mu)}-$ квазимногочлен, где $f_{i} \in \operatorname{Tr} \operatorname{GM}\left(r_{i}, i\right), \theta_{i} \in \Theta$.

Возможны два случая.

1. Существует $j$ такое, что $f_{j}$ зависит от переменных, соответствующих переменной $t$.

Рассмотрим подслучаи:

a) $j \in \sigma$; тогда $C_{t, g}^{\sigma} f=0$, если $\operatorname{deg} g \geqslant 1$, и $C_{t, g}^{\sigma} f=\gamma f$, если $\operatorname{deg} g \leqslant 0$;

b) $j \notin \sigma$; тогда $C_{t, g}^{\sigma} f=f^{(\alpha(t, g))}$.

2. Сушествует $j$ такое, что $y\left(\theta_{j}\right)=t$.

Рассмотрим подслучаи:

a) $j, j^{+} \in \sigma$; тогда $C_{t, g}^{\sigma} f=0$, если $\operatorname{deg} g \geqslant 1$, и $C_{t, g}^{\sigma} f=\gamma f$, если $\operatorname{deg} g \leqslant 0$;

b) $j \in \sigma, j^{+} \notin \sigma$; тогда

$$
C_{t, g}^{\sigma} f=f_{1} \theta_{1} \ldots f_{j} \theta_{j} \lambda_{j+1}(g) f_{j+1} \theta_{j+1} \ldots f_{n} \theta_{n}
$$

если $j<n$, или

$$
C_{t, g}^{\sigma} f=\lambda_{1}(g) f
$$

если $j=n$;

c) $j \notin \sigma, j^{+} \in \sigma ;$ тогда

$$
C_{t, g}^{\sigma} f=f_{1} \theta_{1} \ldots f_{j}\left(\sum_{l=1}^{s} \gamma_{l} \lambda_{j}\left(t y_{1, l} \ldots y_{k-1, l}\right) \theta\left(y_{k, l}\right)\right) f_{j+1} \theta_{j+1} \ldots f_{n} \theta_{n}
$$


если $\operatorname{deg} g \geqslant 1$, и $C_{t, g}^{\sigma} f=\gamma f$, если $\operatorname{deg} g \leqslant 0 ;$

d) $j, j^{+} \notin \sigma$; тогда $C_{t, g}^{\sigma} f=f^{(\alpha(t, g))}$.

Заметим, что $C_{t, 1}^{\sigma}$ - тождественный оператор.

Оператор $D_{t_{1}, \ldots, t_{k}}^{\sigma}$ осуществляет дифференцирование и таким образом помешает переменные, соответствуюшие переменным $t_{1}, \ldots, t_{k}$, на некоторые позиции в квазимногочленах. В свою очередь, оператор $C_{t, g}^{\sigma}$ осушествляет действие на квазимногочлены через переменные, соответствующие переменной $t$.

Пусть $f \in R^{(\sigma, \eta, \xi, \mu)}$ - некоторый квазимногочлен и $g_{1}, \ldots, g_{k} \in \bar{F}$ - однородные многочлены. Выберем различные переменные $t_{1}, \ldots, t_{k} \in S \backslash \mu$. Тогда $E_{g_{1}, \ldots, g_{k}}^{\sigma} f$ определим как квазимногочлен, получающийся из квазимногочлена $C_{t_{1}, g_{1}}^{\sigma} \ldots C_{t_{k}, g_{k}}^{\sigma} D_{t_{1}, \ldots, t_{k}}^{\sigma} f$ заменами $t_{i} \rightarrow 1, i=1, \ldots, k\left(t_{i} \rightarrow 1\right.$ означает замену $\lambda_{j}\left(t_{i}\right) \rightarrow e_{r_{j}}, j=1, \ldots, n$, и $\theta\left(t_{i}\right) \rightarrow 0$, где $e_{r_{j}}$ - единичная матрища размера $r_{j} \times r_{j}$ ). Для произвольного $f \in R(S)$ квазимногочлен $E_{g_{1}, \ldots, g_{k}}^{\sigma} f$ определим по линейности.

Назовем $E_{g_{1}, \ldots, g_{k}}^{\sigma} f$ символической $\sigma$-степенью квазимногочлена $f$ относительно многочленов $g_{1}, \ldots, g_{k}$. Если при этом $\sigma=\sigma_{0}$, то $E_{g_{1}, \ldots, g_{k}}^{\sigma} f$ называется просто символической степенью квазимногочлена $f$.

Введем также понятие символической степени многочлена $f \in \bar{F}$ относительно многочленов $g_{1}, \ldots, g_{k} \in \bar{F}$. Выберем различные переменные $t_{1}, \ldots, t_{k} \in S$. Рассмотрим компоненту $h$ многочлена, получаюшегося из многочлена $f$ заменой $y \rightarrow y\left(1+t_{1}+\cdots+t_{k}\right), y \in G$, имеюшую первую степень по переменным $t_{1}, \ldots, t_{k}$. Тогда через $f^{\left(g_{1}, \ldots, g_{k}\right)}$ обозначим многочлен, получающийся из многочлена $h$ заменой $t_{i} \rightarrow g_{i}, i=1, \ldots, k$.

Отметим следуюшие свойства введенной выше операции символической $\sigma$-степени.

1. Любое выражение вида $E_{1} \ldots E_{k} f$, где $E_{1}, \ldots, E_{k}$ - операторы взятия символической $\sigma$-степени, есть линейная комбинация выражений вида $E f$, где $E$ - оператор взятия символической $\sigma$-степени.

2. Для различных $t_{1}$ и $t_{2}$ из множества $S$ операторы $C_{t_{1}, g}^{\sigma}$ и $C_{t_{2}, h}^{\sigma}$ коммутируют и $C_{t, g_{1}}^{\sigma} C_{t, g_{2}}^{\sigma}=C_{t, g_{1} g_{2}}^{\sigma}$.

3. Любое выражение вида $E_{g_{1}, \ldots, g_{k}}^{\sigma} C_{t, g}^{\sigma} f$, где $f \in R^{(\sigma, \eta, \xi, \mu)}$ и $t \in \mu$, есть линейная комбинация выражений вида $C_{t, h}^{\sigma} E_{h_{1}, \ldots, h_{l}}^{\sigma} f$.

4. Пусть $f \in R^{(\sigma, \eta, \xi, \mu)}$ - некоторьй квазимногочлен и $g_{1}, \ldots, g_{k}, h_{1}, \ldots, h_{l} \in$ $\bar{F}$ - однородные многочлены. Рассмотрим различные переменные $t_{1}, \ldots, t_{l} \in S \backslash \mu$. Тогда квазимногочлен, полученный из квазимногочлена $C_{t_{1}, h_{1}}^{\sigma} \ldots C_{t_{l}, h_{l}}^{\sigma} E_{g_{1}, \ldots, g_{k}}^{\sigma} \times$ $D_{t_{1}, \ldots, t_{l}}^{\sigma} f$ заменами $t_{i} \rightarrow 1, i=1, \ldots, l$, равен линейной комбинации выражений вида $E f$, где $E$ - оператор взятия символической $\sigma$-степени.

Эти свойства легко доказать методами, использованными в работе [8].

Непосредственным следствием предыдуших пунктов является

5. Пусть $f \in R^{(\sigma, \eta, \xi, \mu)}$ - некоторый квазимногочлен. Рассмотрим некоторые операторы $E_{1}, \ldots, E_{k}$, являюшиеся произведениями операторов взятия символических $\sigma$-степеней. Возьмем различные переменные $t_{1}, \ldots, t_{l} \in S \backslash \mu$. Пусть также $C_{0}, \ldots, C_{k}$ - операторы, являюшиеся произведениями операторов вида $C_{t_{i}, h}^{\sigma}$. Тогда квазимногочлен, полученный из квазимногочлена $C_{0} E_{1} C_{1} \ldots C_{k-1} E_{k} C_{k} \times$ $D_{t_{1}, \ldots, t_{l}}^{\sigma} f$ заменами $t_{i} \rightarrow 1, i=1, \ldots, l$, равен линейной комбинации выражений 
вида $E f$, где $E$ - оператор взятия символической $\sigma$-степени.

Основным техническим моментом при доказательстве свойств 1-5 являются следуюшие предложения.

ПРЕДЛОЖЕнИЕ 1. Пусть $f \in R^{(\sigma, \eta, \xi, \mu)}$ - некоторый однородный квазимногочлен. Предположим, что $f$ зависит только от одной переменной у из $G$ u имеет по ней первую степень. Пусть $t \notin \mu u g, h \in \bar{F}$ - однородные многоилены. Тогда квазимногочлен, получающийся из $C_{t, h}^{\sigma} E_{g}^{\sigma} D_{t}^{\sigma} f$ заменой $t \rightarrow 1$, равен $E_{g h}^{\sigma} f$.

ПРЕДЛОЖЕНИЕ 2. Пусть $f \in R^{(\sigma, \eta, \xi, \mu)}-$ некоторьй однородный квазимногочлен. Предположим, что $f$ не зависит ни от одной переменной из G. Пусть $t \in \mu u g_{1}, \ldots, g_{k}, h \in \bar{F}$ - однородные многочлены. Тогда $E_{g_{1}, \ldots, g_{k}}^{\sigma} C_{t, h}^{\sigma} f=C_{t, h^{\left(g_{1}, \ldots, g_{k}\right)}}^{\sigma}$.

3. Ширина квазимногочленов. Пусть имеется однородный квазимногочлен степени $a$ вида

$$
f=f_{1} \theta_{1} \ldots f_{n} \theta_{n} \in R(S),
$$

где $f_{i} \in \operatorname{Tr} \operatorname{GM}\left(r_{i}, i\right), \theta_{i} \in \Theta$.

Тогда положим $b_{\sigma}(f)=a-\sum_{j \in \sigma} \operatorname{deg}_{G} f_{j}$. Это число назовем $\sigma$-шириной квазимногочлена $f$ (см. [8]). Произвольный однородньй элемент $g \in R(S)$ можно представить в виде несократимой суммы: $g=g_{1}+\cdots+g_{k}$, где каждое $g_{i}$ имеет вид (12).

Тогда положим

$$
b_{\sigma}(g)=\max _{i=1, \ldots, k} b_{\sigma}\left(g_{i}\right), \quad p_{\sigma}(g)=\sum\left\{g_{i}: b_{\sigma}\left(g_{i}\right)=b_{\sigma}(g)\right\} .
$$

Квазимногочлен $p_{\sigma}(g)$ назовем $\sigma$-старшей частью $g$. Однородный квазимногочлен $g$, являющийся суммой квазимногочленов вида (12), имеющих одинаковую $\sigma$-ширину $k$, называется $\sigma$-однородным квазимногочленом. Число $k$ при этом называется $\sigma$-шириной $g$. Естественным образом вводится понятие $\sigma$-однородной компоненты.

Введенное вьше понятие символической $\sigma$-степени связано с понятием $\sigma$-ширины следуюшим образом. Пусть $f$ есть $\sigma$-однородный квазимногочлен и $\sigma^{\prime} \subset \sigma$. Предположим также, что все квазимногочлены $g_{1}, \ldots, g_{k} \in \bar{F}$ имеют степень, большую нуля. Тогда квазимногочлен $E_{g_{1}, \ldots, g_{k}}^{\sigma} f$ равен $\sigma$-однородной компоненте квазимногочлена $E_{g_{1}, \ldots, g_{k}}^{\sigma^{\prime}} f$, имеющей $\sigma$-ширину, равную $\sigma$-ширине $f$. При этом если $E_{g_{1}, \ldots, g_{k}}^{\sigma} f$ не равен нулю, то $E_{g_{1}, \ldots, g_{k}}^{\sigma} f=p_{\sigma}\left(E_{g_{1}, \ldots, g_{k}}^{\sigma^{\prime}} f\right)$.

Подпространство $M$ пространства $R(S)$ назовем $\sigma$-однородным, если оно однородно в обычном смысле и любая $\sigma$-однородная компонента любого квазимногочлена из $M$ принадлежит $M$.

$\sigma$-однородное подпространство пространства $R(S)$, замкнутое относительно взятия символических $\sigma$-степеней, назовем $\left(T_{2}, \sigma\right)$-пространством.

Пусть $M$ - некоторое однородное подпространство пространства $R(S)$. Тогда через $\Delta_{\sigma}(M)$ обозначим пространство, натянутое на элементы $p_{\sigma}(f)$, где $f$ - однородный многочлен из $M$. Легко видеть, что $\Delta_{\sigma}(M) \sigma$-однородно.

Справедлива 
Лемма 13. Пусть $M \subset R(S)$ есть $\left(T_{2}, \sigma^{\prime}\right)$-пространство и $\sigma^{\prime} \subset \sigma$. Тогда $\Delta_{\sigma}(M)$ есть $\left(T_{2}, \sigma\right)$-пространство.

ДокАЗАТЕЛЬСтво. Пусть $g_{1}, \ldots, g_{k} \in \bar{F}$ - однородные многочлены. Без ограничения обшности можно считать, что $g_{1}, \ldots, g_{l}$ суть константы и $\operatorname{deg} g_{l+1}, \ldots$ $\ldots, \operatorname{deg} g_{k} \geqslant 1$. Пусть $f \in M$ - однородный квазимногочлен. Нам достаточно показать, что $E_{g_{1}, \ldots, g_{k}}^{\sigma} p_{\sigma}(f) \in \Delta_{\sigma}(M)$. Учитывая, что квазимногочлен $p_{\sigma}(f)$ $\sigma$-однороден, получим, что $E_{g_{1}, \ldots, g_{k}}^{\sigma_{j}} p_{\sigma}(f)=\alpha E_{g_{l+1}, \ldots, g_{k}}^{\sigma} p_{\sigma}(f)$, где $\alpha \in K$.

Теперь легко проверить, что либо $E_{g_{l+1}, \ldots, g_{k}}^{\sigma} p_{\sigma}(f)=0$, либо

$$
E_{g_{l+1}, \ldots, g_{k}}^{\sigma} p_{\sigma}(f)=p_{\sigma}\left(E_{g_{l+1}, \ldots, g_{k}}^{\sigma^{\prime}} p_{\sigma}(f)\right)=p_{\sigma}\left(E_{g_{l+1}, \ldots, g_{k}}^{\sigma^{\prime}} f\right)
$$

Лемма доказана.

4. Построение пространств $L^{(\sigma, \eta)}$. Фиксируем попарно различные переменные $\left\{t_{j, i}: j=1, \ldots, n, i=1, \ldots, n+1\right\} \subset S$. Пусть $S_{q}=\left\{t_{j, i}: j \leqslant q, i \leqslant n+1\right\}$.

Пусть теперь $L \subset R(\varnothing)$ - произвольное $\left(T_{2}, \sigma_{0}\right)$-пространство. Построим по индукции некоторую систему подпространств

$$
L^{(\sigma, \eta)} \subset+\left\{R^{\left(\sigma, \eta, \xi, S_{p}\right)}: \xi \subset S_{p}, \xi \cap \bar{\eta}=\varnothing\right\},
$$

где $p=|\sigma|$ и $\left|\bar{\eta} \cap\left\{t_{k, i}: i=1, \ldots, n+1\right\}\right|=1$ для $k=1, \ldots, p$. Набор $\eta$, удовлетворяющий этому свойству, назовем допустимым, и пару $(\sigma, \eta)$ также назовем допустимой.

Положим $L^{\left(\sigma_{0}, \eta_{0}\right)}=L$. Предположим, что $L^{(\sigma, \eta)}$ уже построено и что $|\sigma|=$ $q-1<n$.

Пусть

$$
B=D_{t_{q, 1}, \ldots, t_{q, n+1}}^{\sigma} .
$$

Через $C L^{(\sigma, \eta)}$ обозначим замыкание пространства $B L^{(\sigma, \eta)}$ относительно взятия операций $C_{t_{q, i}, g}^{\sigma}$, где $i=1, \ldots, n+1$ и $g \in \bar{F}$, и взятия символических $\sigma$-степеней.

Пусть $A_{0}=\{1, \ldots, n\} \backslash \sigma$. Через $A$ обозначим множество пар $(i, j)$, где $j \in A_{0}$ и $i=1, \ldots, n+1$.

Положим по определению

$$
K_{i, j}=+\left\{R^{\left(\sigma_{j}, \eta_{i, j}, \xi, S_{q}\right)}: \exists \xi \subset S_{q}, \xi \cap \bar{\eta}=\varnothing, t_{q, i} \notin \xi, t_{q, i+1}, \ldots, t_{q, n+1} \in \xi\right\},
$$

где набор $\eta_{i, j}$ получается из набора $\eta$ вставкой переменной $t_{q, i}$ между местом $m(\sigma, j)-1$ и местом $m(\sigma, j)$ слева.

Тогда имеем

$$
C L^{(\sigma, \eta)} \subset K, \quad K=\bigoplus_{(i, j) \in A} K_{i, j}
$$

Считаем, что $\left(i_{1}, j_{1}\right)<\left(i_{2}, j_{2}\right)$, если первая пара меньше второй лексикографически. Через $P_{i, j}$ обозначим проектор пространства $K$ на $K_{i, j}$. 
Положим

$$
C_{i, j} L^{(\sigma, \eta)}=P_{i, j}\left(C L^{(\sigma, \eta)} \cap \bigoplus_{\substack{(i, j) \leqslant\left(i^{\prime}, j^{\prime}\right) \\\left(i^{\prime}, j^{\prime}\right) \in A}} K_{i^{\prime}, j^{\prime}}\right) .
$$

Легко видеть, что пространство $C_{i, j} L^{(\sigma, \eta)}$ замкнуто относительно действия операторов $C_{t_{q, i}, g}^{\sigma}, g \in \bar{F}$, а также относительно взятия символических $\sigma$-степеней. Действительно, пространство, стояшее под знаком оператора $P_{i, j}$ в формуле, определяюшей $C_{i, j} L^{(\sigma, \eta)}$, и пространства $K_{i, j}$, где $(i, j) \leqslant\left(i^{\prime}, j^{\prime}\right)$, замкнуты относительно этих операций. Наконец, положим $L^{\left(\sigma_{j}, \eta_{i, j}\right)}=\Delta_{\sigma_{j}}\left(C_{i, j} L^{(\sigma, \eta)}\right)$.

По лемме 13 если $L^{(\sigma, \eta)}$ есть $\left(T_{2}, \sigma\right)$-пространство, то $L^{\left(\sigma_{j}, \eta_{i, j}\right)}$ есть $\left(T_{2}, \sigma_{j}\right)$-пространство. Кроме того, $L^{\left(\sigma_{j}, \eta_{i, j}\right)}$, как и $C_{i, j} L^{(\sigma, \eta)}$, замкнуто относительно действия эндоморфизмов $\alpha\left(t_{q, i}, g\right)$, где $g \in \bar{F}$.

Отсюда получаем, что справедлива

Лемма 14. Пусть $L \subset R(\varnothing)$ есть $\left(T_{2}, \sigma_{0}\right)$-пространство и $\eta=\left(t_{1}, \ldots, t_{q}\right)$. Тогда пространство $L^{(\sigma, \eta)}$, построенное описанным выше способом, есть $\left(T_{2}, \sigma\right)$-пространство, замкнутое относительно действия әндоморфизмов $\alpha\left(t_{i}, g\right)$, әде $i=1, \ldots, q u g \in \bar{F}$.

Пусть $J_{i}$ - некоторый $T_{2}$-идеал алгебры $\operatorname{Tr} \operatorname{GM}\left(r_{i}, i\right), \quad i=1, \ldots, n$. Через $I\left(J_{1}, \ldots, J_{n}\right)$ обозначим подпространство пространства $R(S)$, натянутое на квазимногочлены вида $h_{1} f_{1} h_{1}^{\prime} \theta_{1} \ldots h_{n} f_{n} h_{n}^{\prime} \theta_{n}$, где $f_{i} \in \operatorname{Tr}_{G_{1}}(\varnothing) \cap J_{i}, h_{i}, h_{i}^{\prime} \in$ $\operatorname{Tr}_{\mathrm{GM}_{i}}(S), \theta_{i} \in \Theta$. Заметим, что $T_{2}$-идеалы $J_{i}$ однородные в силу бесконечности поля $K$.

Докажем следующее утверждение, устанавливающее связь между $L$ и пространствами $L^{(\sigma, \eta)}$.

Лемма 15. Пусть $L, L_{1} u L_{2} \subset R(\varnothing)$ есть $\left(T_{2}, \sigma_{0}\right)$-пространства. Тогда справедливы следующие утверждения:

1) если $L_{1} \subset L_{2}$, mo $L_{1}^{(\sigma, \eta)} \subset L_{2}^{(\sigma, \eta)}$;

2) $L_{1}^{(\sigma, \eta)}+L_{2}^{(\sigma, \eta)} \subset\left(L_{1}+L_{2}\right)^{(\sigma, \eta)}$;

3) пусть $L_{1} \subset L_{2}$ и для любой допустимой пары $(\sigma, \eta)$ такой, что $|\sigma|=$ $|\eta|=n$, выполнено $L_{1}^{(\sigma, \eta)}=L_{2}^{(\sigma, \eta)} ;$ тогда $L_{1}=L_{2}$;

4) если $L \subset I\left(J_{1}^{s_{1}}, \ldots, J_{n}^{s_{n}}\right)$, mo $L^{(\sigma, \eta)} \subset I\left(J_{1}^{s_{1}-r}, \ldots, J_{n}^{s_{n}-r}\right), \quad r=|\sigma|(n+1)$.

ДокАЗАТЕЛЬство. Утверждение 1) очевидно по построению.

$2)$ В силу п. 1) получаем $L_{1}^{(\sigma, \eta)} \subset\left(L_{1}+L_{2}\right)^{(\sigma, \eta)}$ и $L_{2}^{(\sigma, \eta)} \subset\left(L_{1}+L_{2}\right)^{(\sigma, \eta)}$. Из того, что $\left(L_{1}+L_{2}\right)^{(\sigma, \eta)}$ есть линейное пространство, получаем требуемое утверждение.

3) Воспользуемся здесь системой обозначений, введенной при построении пространств $L^{\left(\sigma_{j}, \eta_{i, j}\right)}$. Достаточно показать, что если для всех $(i, j) \in A$ выполнено $L_{1}^{\left(\sigma_{j}, \eta_{i, j}\right)}=L_{2}^{\left(\sigma_{j}, \eta_{i, j}\right)}$, то $L_{1}^{(\sigma, \eta)}=L_{2}^{(\sigma, \eta)}$. Стандартные рассуждения о старших частях и фильтрациях показьвают, что $C_{i, j} L_{1}^{(\sigma, \eta)}=C_{i, j} L_{2}^{(\sigma, \eta)}$. Аналогичные рассуждения дают $C L_{1}^{(\sigma, \eta)}=C L_{2}^{(\sigma, \eta)}$. 
Пусть $f \in L_{2}^{(\sigma, \eta)}-\sigma$-однородный квазимногочлен. Тогда $B f \in B L_{2}^{(\sigma, \eta)} \subset$ $C L_{2}^{(\sigma, \eta)}=C L_{1}^{(\sigma, \eta)}$. Пусть $g-$ квазимногочлен, получаюшийся из $B f$ в результате подстановки $t_{|\sigma|+1, i} \rightarrow 1, \quad i=1, \ldots, n+1$. Учитывая, что $L_{1}^{(\sigma, \eta)}$ есть $\left(T_{2}, \sigma\right)$-пространство (см. [9], унитарный принцип), получаем $g \in L_{1}^{(\sigma, \eta)}$. С другой стороны, непосредственное вычисление с учетом $\sigma$-однородности $f$ дает $g=\alpha f$, где $\alpha$ - ненулевой элемент $K$. Здесь для того, чтобы заключить, что $\alpha \neq 0$, нужно воспользоваться фактом char $K=0$. Тогда $f \in L_{1}^{(\sigma, \eta)}$ и $L_{1}^{(\sigma, \eta)}=L_{2}^{(\sigma, \eta)}$.

4) Так как $L^{(\sigma, \eta)} \subset R\left(S_{q}\right)$, где $q=|\sigma|$ и $\left|S_{q}\right|=r=q(n+1)$, то максимальное количество "испорченных" элементов из идеалов $J_{i}$ не превзойдет $r$. Тот факт, что элементы $T_{2}$-идеалов $J_{i}$ не разрушаются при взятии операции $\Delta_{\sigma^{\prime}}$, следует из вьшеупомянутой однородности $J_{i}$. Лемма доказана.

\section{§7. Абстрактное линейное следование}

1. Общая теория. Пусть $V$-произвольный модуль над коммутативным кольцом $\Phi$ с единицей. Предположим, что у нас имеется некоторое отношение "следования" $\rightarrow$, которое применяется в виде $U \rightarrow a$, где $U$ - подмодуль модуля $V$ и $a \in V$. Если $U$ и $W-$ подмодули модуля $V$ и $U \rightarrow w$ для любого $w \in W$, то считаем, что $U \rightarrow W$.

Предположим, что отношение $\rightarrow$ удовлетворяет следуюшим условиям:

1) если $U \rightarrow W$ и $W \rightarrow a$, то $U \rightarrow a$;

2) если $U_{1} \subset U_{2}$ и $U_{1} \rightarrow a$, то $U_{2} \rightarrow a$;

3) если $a \in V$, то $V \rightarrow a$;

4) если $U_{1} \rightarrow a_{1}, \ldots, U_{k} \rightarrow a_{k}$ и $\alpha_{1}, \ldots, \alpha_{k} \in \Phi$, то $U_{1}+\cdots+U_{k} \rightarrow \alpha_{1} a_{1}+\cdots$ $\cdots+\alpha_{k} a_{k}$.

Непосредственным следствием п. 4) является

5) если $U \rightarrow a+b$ и $U \rightarrow b$, то $U \rightarrow a$.

Здесь мы не обсуждаем независимости аксиоматики 1)-4).

Пусть $S$ - подмножество элементов $V$. Через $S_{\Phi}$ обозначим подмодуль модуля $V$, порожденный множеством $S$ и нулем. Таким образом, $\varnothing_{\Phi}=0$.

Пусть $U$ - некоторый подмодуль в $V$. Считаем, что последовательность $v_{1}, \ldots$ $\ldots, v_{i}, \ldots$ элементов $V$ является конечно базируемой по модулю $U$, если сушествует некоторое $m$ такое, что для всех $i>m$ выполнено $\left\{v_{1}, \ldots, v_{m}\right\}_{\Phi} \rightarrow v_{i}+u_{i}$ для некоторого $u_{i} \in U$. Само множество $\left\{v_{1}, \ldots, v_{m}\right\}$ называется базой всей последовательности по модулю $U$. В случае $U=0$ сохраняем те же названия, только опускаем выражение "по модулю $U$ ".

Пусть теперь $V=V_{1} \supset \cdots \supset V_{n} \supset \cdots$-некоторая счетная фильтрация. Последовательность $v_{1}, \ldots, v_{i}, \ldots$ назовем бесконечно погружающейся относительно данной фильтрации, если для любого $i$ выполнено $v_{i} \in V_{n_{i}}$ и $\lim _{i \rightarrow \infty} n_{i}=\infty$.

Имеет место

ЛЕмма 16. Пусть $v_{1}, \ldots, v_{i}, \ldots$ - последовательность, конечно базируемая по модулю любого $V_{n}$. Пусть также любая бесконечно погружающаяся относительно фильтрации $V=V_{1} \supset \cdots \supset V_{n} \supset \cdots$ последовательность конечно базируема. Тогда исходная последовательность $v_{1}, \ldots, v_{i}, \ldots$ конечно базируема. 
ДоКАЗАТЕЛЬСТВо. Построим элементы $w_{i, j} \in V_{i}$, где $j \geqslant m_{i}$, такие, что

$$
\left\{v_{1}, \ldots, v_{m_{i}-1}\right\}_{\Phi} \rightarrow v_{j}+w_{i, j} \text { для } j \geqslant m_{i},
$$

где $i_{1}<i_{2} \Longrightarrow m_{i_{1}}<m_{i_{2}}$. Считаем также, что $m_{1}=1$.

Диагональная последовательность

$$
w_{1,1}, \ldots, w_{1, m_{2}-1}, \ldots, w_{k, m_{k}}, \ldots, w_{k, m_{k+1}-1}, \ldots
$$

является бесконечно погружающейся и поэтому конечно базируема.

Пусть $S=\left\{w_{i, j}: i \leqslant r, m_{i} \leqslant j \leqslant m_{i+1}-1\right\}$ - база этой последовательности и $U=S_{\Phi}$. Из формулы (13) получаем $\left\{v_{1}, \ldots, v_{m_{i}-1}, v_{j}\right\}_{\Phi} \rightarrow w_{i, j}$ для $j \geqslant m_{i}$. Тогда справедливо соотношение $V=\left\{v_{1}, \ldots, v_{m_{r+1}-1}\right\}_{\Phi} \rightarrow U$.

Докажем индукцией по $i \geqslant r+1$ следующее

УТВЕРЖДЕНИЕ $2 . V \rightarrow v_{j}$ для $m_{i} \leqslant j \leqslant m_{i+1}-1$.

ДокАЗАТЕЛЬСТво. База индукции: $i=r+1$. Пусть $m_{r+1} \leqslant j \leqslant m_{r+2}-1$. Так как $w_{i, j} \in U$, то $V \rightarrow w_{i, j}$. По формуле (13) имеем $V \rightarrow v_{j}+w_{i, j}$. Тогда $V \rightarrow v_{j}$.

Предположим, что для некоторого $i \geqslant r+1$ утверждение выполнено и $m_{i+1} \leqslant$ $k \leqslant m_{i+2}-1$. По формуле (13) имеем $V^{\prime}=\left\{v_{1}, \ldots, v_{m_{i+1}-1}\right\}_{\Phi} \rightarrow v_{k}+w_{i+1, k}$. По предположению индукции $V \rightarrow V^{\prime}$ и $V \rightarrow v_{k}+w_{i+1, k}$. Так как $w_{i+1, k} \in U$, то $V \rightarrow w_{i+1, k}$ и $V \rightarrow v_{k}$. Утверждение доказано.

Из свойства 3 ) получаем $V \rightarrow v_{j}$ для $j \leqslant m_{r+1}-1$. Таким образом, множество $\left\{v_{1}, \ldots, v_{m_{r+1}-1}\right\}$ есть база последовательности $v_{1}, \ldots, v_{n}, \ldots$ Лемма доказана.

Из этого утверждения получаем

СлЕДСТВИЕ 2. Пусть отношение $\rightarrow$ удовлетворяет условиям:

а) любая последовательность конечно базируема по модулю $V_{n}$ для любо$20 \mathrm{n}$;

b) любая бесконечно погружающаяся относительно фильтрации $V=$ $V_{1} \supset \cdots \supset V_{n} \supset \cdots$ последовательность конечно базируема.

Тогда любая последовательность конечно базируема.

2. Один частный случай. Применим результаты предыдущего пункта к некоторому частному случаю, который не будет использоваться в дальнейшем.

Пусть $A=Z\left\langle x_{1}, \ldots, x_{n}, \ldots\right\rangle$ и $A_{m}=Z_{m}\left\langle x_{1}, \ldots, x_{n}, \ldots\right\rangle$ - свободные ассоциативные алгебры над кольцом целых чисел $Z$ и кольцом $Z_{m}$ вычетов по модулю $m$ соответственно. Пусть также $\varphi_{m}: A \rightarrow A_{m}$ - естественная проекция.

В работе [10] А.В. Гришин поставил следуюший вопрос: верно ли, что любая последовательность многочленов $f_{1}, \ldots, f_{i}, \ldots \in A$ такая, что для любого числа $m \in \mathbb{N}$ последовательность $\varphi_{m}\left(f_{1}\right), \ldots, \varphi_{m}\left(f_{i}\right), \ldots$ порождает конечно базируемое $T$-пространство в $A_{m}$, порождает конечно базируемое $T$-пространство в $A$ ?

Определим отношение $\rightarrow$ следуюшим образом: $U \rightarrow v$, где $U$ есть $Z$-подмодуль в $A$ и $v \in A$, если $v$ принадлежит $T$-пространству, порожденному пространством $U$. Легко видеть, что условия 1)-5) выполнены. 
Рассмотрим цепочку чисел $m_{1}, \ldots, m_{i}, \ldots$ такую, что $m_{1}=1$, при $i_{1}<i_{2}$ число $m_{i_{2}}$ делится на $m_{i_{1}}$ и для любого простого числа $p$ показатель при $p$ в разложении $m_{i}$ на простые числа стремится к бесконечности, когда $i$ стремится к бесконечности. Очевидно, такие последовательности существуют.

Фиксируем фильтрацию $V_{i}=m_{i} A, i \in \mathbb{N}$. Пусть имеется последовательность $f_{1}, \ldots, f_{i}, \ldots \in A$ такая, что для любого числа $m \in \mathbb{N}$ последовательность $\varphi_{m}\left(f_{1}\right), \ldots, \varphi_{m}\left(f_{i}\right), \ldots$ порождает конечно базируемое $T$-пространство. Лемма 16 показывает, что для того чтобы сказать, что исходная последовательность порождает конечно базируемое $T$-пространство, достаточно установить, что любая бесконечно погружаюшаяся относительно фильтрации $V_{1} \supset \cdots \supset V_{n} \supset \cdots$ последовательность $g_{1}, \ldots, g_{i}, \ldots$ конечно базируема.

Но последовательность $g_{1}, \ldots, g_{i}, \ldots$ бесконечно погружающаяся относительно фильтрации $V_{1} \supset \cdots \supset V_{n} \supset \cdots$ тогда и только тогда, когда для любого простого числа $p$ и произвольного натурального числа $k$ в последовательности $\varphi_{p^{k}}\left(f_{1}\right), \ldots, \varphi_{p^{k}}\left(f_{i}\right), \ldots$ существует лиш конечное число членов, отличных от нуля.

Таким образом, мы получаем следующую эквивалентную переформулировку проблемы А.В Гришина: верно ли, что любая последовательность многочленов $f_{1}, \ldots, f_{i}, \ldots \in A$ такая, что для любого простого числа $p$ и произвольного натурального числа $k$ в последовательности $\varphi_{p^{k}}\left(f_{1}\right), \ldots, \varphi_{p^{k}}\left(f_{i}\right), \ldots$ существует лишь конечное число членов, отличных от нуля, порождает конечно базируемое $T$-пространство в $A$ ?

Все сказанное в этом пункте верно, если выражение “T-пространство” заменить на выражение "Т-идеал". Заметим, что примеры из работ [4], [5], [10], [11] показывают, что не любая цепочка многочленов из $A$ порождает конечно базируемое $T$-пространство ( $T$-идеал). Однако природа этих примеров такова, что существует некоторое простое $p$ такое, что проекции этих цепочек на $A_{p}$ порождают бесконечно базируемые $T$-пространства ( $T$-идеалы). Таким образом, поставленньй выше вопрос пока открыт.

\section{§ 8. Доказательство основного результата}

В этом параграфе мы используем лемму Артина-Рисса [14], способ применения которой к доказательству конечной базируемости был замечен А. Я. Беловым.

Применим теперь следствие 2 к случаю, когда $V=R(\varnothing)$, а $U \rightarrow f$ тогда и только тогда, когда $f$ принадлежит $T_{2}$-пространству алгебры $F$, порожденному $U$, где $U$ - линейное подпространство в $R(\varnothing)$ и $f \in R(\varnothing)$.

Легко проверить, что для таким образом определенного “следования" условия 1)-5) выполнены.

Для $i=1, \ldots, n$ и $r_{i} \geqslant 2$ через $C_{i}$ обозначим $T_{2}$-идеал алгебры $\operatorname{Tr} \operatorname{GM}_{i}\left(r_{i}, i\right)$, порожденный элементами

$$
\begin{gathered}
\operatorname{tr}\left(c_{r_{i}^{2}}\left(u_{1}, \ldots, u_{r_{i}^{2}}, v_{1}, \ldots, v_{r_{i}^{2}}\right)\right) \\
\operatorname{tr}\left(c_{r_{i}^{2}}\left(u_{1}^{\prime}, \ldots, u_{r_{i}^{2}}^{\prime}, v_{1}^{\prime}, \ldots, v_{r_{i}^{2}}^{\prime}\right)\right) \operatorname{tr}\left(c_{r_{i}^{2}}\left(u_{1}^{\prime \prime}, \ldots, u_{r_{i}^{2}}^{\prime \prime}, v_{1}^{\prime \prime}, \ldots, v_{r_{i}^{2}}^{\prime \prime}\right)\right),
\end{gathered}
$$


где $u_{i}, u_{i}^{\prime}, u_{i}^{\prime \prime}, v_{i}, v_{i}^{\prime}, v_{i}^{\prime \prime} \in \mathrm{GM}_{i}\left(r_{i}, i\right)$ - однородные элементы и $\operatorname{deg}_{Z} u_{1} \ldots u_{r_{i}^{2}} v_{1} \ldots$ $\ldots v_{r_{i}^{2}}, \operatorname{deg}_{Z} u_{1}^{\prime} \ldots u_{r_{i}^{2}}^{\prime} v_{1}^{\prime} \ldots v_{r_{i}^{2}}^{\prime}+\operatorname{deg}_{Z} u_{1}^{\prime \prime} \ldots u_{r_{i}^{2}}^{\prime \prime} v_{1}^{\prime \prime} \ldots v_{r_{i}^{2}}^{\prime \prime}$ суть четные числа. В случае $r_{i}=1$ положим $C_{i}=\operatorname{Tr} \operatorname{GM}(1, i)$.

В этих формулах $c_{r_{i}^{2}}$ означает полином Капелли, и все эти определения корректны, так как полиномы (14) и (15) суть центральные полиномы Размыслова (см., например, [15]).

Пусть $U_{m}=I\left(C_{1}^{m}, \ldots, C_{n}^{m}\right) \cap R(\varnothing)$. Фиксируем фильтрацию

$$
R(\varnothing) \supset U_{1} \supset \cdots \supset U_{i} \supset \cdots
$$

и применим следствие 2 .

Проверим условие а). Имеет место

Лемма 17. Пусть $\bar{C}_{i}-$ идеал алгебры $\operatorname{Tr} \mathrm{GM}_{i}(\varnothing)$, порожденный әлементами $\operatorname{tr}\left(c_{r_{i}^{2}}\left(u_{1}, \ldots, u_{r_{i}^{2}}, v_{1}, \ldots, v_{r_{i}^{2}}\right)\right)$, где $u_{i}, v_{i} \in \mathrm{GM}_{i}(\varnothing)$. Тогда для любого $\mathrm{m}$ имеем $\bar{C}_{i}^{2 m} \subset C_{i}^{m}$.

ДокАЗАТЕльСтво. Пусть $f=\prod_{j=1}^{2 m} f_{j}$, где $f_{j}=\operatorname{tr}\left(c_{r_{i}^{2}}\left(u_{j, 1}, \ldots, u_{j, r_{i}^{2}}, v_{j, 1}, \ldots\right.\right.$ $\left.\left.\ldots, v_{j, r_{i}^{2}}\right)\right)$ - однородный квазимногочлен и $u_{j, i}, v_{j, i} \in \mathrm{GM}(\varnothing)$. В силу коммутируемости следов с общими матрищами такие элементы $f$ порождают $\bar{C}_{i}^{2 m}$ как идеал. По крайней мере одно из чисел $\operatorname{deg}_{Z} f_{j}, \operatorname{deg}_{Z} f_{j+1}, \operatorname{deg}_{Z} f_{j}+\operatorname{deg}_{Z} f_{j+1}$ четное. Тогда хотя бы один элемент $f_{j}, f_{j+1}, f_{j} f_{j+1}$ имеет вид (14) или (15). Отсюда $f_{j} f_{j+1} \in C_{i}$ и $f \in C_{i}^{m}$. Лемма доказана.

Эта лемма показывает, что алгебра $\operatorname{Tr} \mathrm{GM}_{i}(\varnothing) / C_{i}^{m} \cap \operatorname{Tr} \mathrm{GM}_{i}(\varnothing)$ имеет матричную сложность строго меньше, чем $r_{i}$.

Назовем индексом набора $r_{1}, \ldots, r_{n}$ бесконечный набор ind $\left(r_{1}, \ldots, r_{n}\right)=\left(s_{2}\right.$, $\left.s_{3}, \ldots\right)$, где $s_{i}$ - количество чисел из набора $r_{1}, \ldots, r_{n}$, равных $i$. Два индекса будем сравнивать антилексикографически.

Для того чтобы доказать конечную базируемость любой цепочки элементов из $R(\varnothing)$ относительно отношения $\rightarrow$ по модулю $U_{m}$, достаточно доказать конечную базируемость любой цепочки по модулю каждого пространства

$$
\begin{aligned}
& I\left(\operatorname{Tr} \operatorname{GM}\left(r_{1}, 1\right), \ldots, \operatorname{Tr} \operatorname{GM}\left(r_{i-1}, i-1\right), C_{i}^{m},\right. \\
& \left.\quad \operatorname{Tr} \operatorname{GM}\left(r_{i+1}, i+1\right), \ldots, \operatorname{Tr} \operatorname{GM}\left(r_{n}, n\right)\right) \cap R(\varnothing) .
\end{aligned}
$$

В случае $r_{i}=1$ это очевидно, поэтому можно считать, что $r_{i} \geqslant 2$. Следовательно, мы должны доказать конечную базируемость любого ограниченного $G$-зависяшего $T_{2}$-пространства в

$$
\begin{gathered}
R\left\langle\operatorname{Tr} \operatorname{GM}\left(r_{1}, 1\right), \lambda_{1}, \ldots, \operatorname{Tr} \operatorname{GM}\left(r_{i-1}, i-1\right), \lambda_{i-1}, \operatorname{Tr} \operatorname{GM}\left(r_{i}, i\right) / C_{i}^{m}, \bar{\lambda}_{i},\right. \\
\left.\operatorname{Tr} \operatorname{GM}\left(r_{i+1}, i+1\right), \lambda_{i+1}, \ldots, \operatorname{Tr} \operatorname{GM}\left(r_{n}, n\right), \lambda_{n}\right\rangle,
\end{gathered}
$$

где $\bar{\lambda}_{i}$ - отображение, индуцированное отображением $\lambda_{i}$.

Эпиморфизм $\bar{\lambda}_{i}: F \rightarrow \operatorname{Tr} \operatorname{GM}\left(r_{i}, i\right) / C_{i}^{m}$ задан формулами $x_{j} \rightarrow x_{j}^{(i)}+C_{i}^{m}$, $z_{j} \rightarrow z_{j}^{(i)}+C_{i}^{m}$, где общие матрищы $x_{j}^{(i)}$ и $z_{j}^{(i)}$ имеют размер $r \times r$. 
Пусть $J$-ядро этого эпиморфизма, а $\xi(J)$-проекция $F$ на $F / J$. Гомоморфизм $\bar{\lambda}_{i}$ с учетом утверждения 1 сводит задачу к доказательству конечной базируемости любого ограниченного $G$-зависящего $T_{2}$-пространства в

$$
\begin{gathered}
R\left\langle\operatorname{Tr} \operatorname{GM}\left(r_{1}, 1\right), \lambda_{1}, \ldots, \operatorname{Tr} \operatorname{GM}\left(r_{i-1}, i-1\right), \lambda_{i-1}, F / J, \xi(J),\right. \\
\left.\operatorname{Tr} \operatorname{GM}\left(r_{i+1}, i+1\right), \lambda_{i+1}, \ldots, \operatorname{Tr} \operatorname{GM}\left(r_{n}, n\right), \lambda_{n}\right\rangle .
\end{gathered}
$$

Существует некоторое число $m^{\prime}$ такое, что $M_{r_{i}-1}^{m^{\prime}} \cap K\langle G\rangle \subset J$. Тогда можно ограничиться доказательством конечной базируемости ограниченных $G$-зависящих $T_{2}$-пространств

B

$$
\begin{aligned}
& R\left\langle\operatorname{Tr} \operatorname{GM}\left(r_{1}, 1\right), \lambda_{1}, \ldots, \operatorname{Tr} \operatorname{GM}\left(r_{i-1}, i-1\right), \lambda_{i-1}, F / M_{r_{i}-1}^{m^{\prime}}, \xi\left(M_{r_{i}-1}^{m^{\prime}}\right),\right. \\
& \left.\operatorname{Tr} \operatorname{GM}\left(r_{i+1}, i+1\right), \lambda_{i+1}, \ldots, \operatorname{Tr} \operatorname{GM}\left(r_{n}, n\right), \lambda_{n}\right\rangle
\end{aligned}
$$

где $\xi\left(M_{r_{i}-1}^{m^{\prime}}\right)$ - проекция $F$ на $M_{r_{i}-1}^{m^{\prime}}$.

Рассмотрим теперь $F$ как подмножество в $Q\left(r_{i}-1\right)$. По лемме 11 имеем $M_{r_{i}-1}^{m^{\prime}}=$ $F \cap \Theta_{m^{\prime}}$. Это позволяет вложить $F / M_{r_{i}-1}^{m^{\prime}}$ в $Q\left(r_{i}-1\right) / \Theta_{m^{\prime}}$. Устраивая конечную фильтрацию по $\theta$-степеням, приходим к проблеме конечной базируемости цепочек элементов относительно аналогично определенного отношения $\rightarrow$ в пространствах

$$
R\langle r_{1}, \ldots, r_{i-1}, \underbrace{r_{i}-1, \ldots, r_{i}-1}_{\text {не более } m^{\prime} \text { раз }}, r_{i+1}, \ldots, r_{n}\rangle .
$$

Индекс набора, стоящего в скобках последнего выражения, строго меньше, чем $\operatorname{ind}\left(r_{1}, \ldots, r_{n}\right)$. Таким образом, используя это рассуждение, получим индукцию по индексу. Если $\operatorname{ind}\left(r_{1}, \ldots, r_{n}\right)=(0,0, \ldots)$, то $r_{1}=\cdots=r_{n}=1$, и тогда $I\left(C_{1}^{m}, \ldots, C_{n}^{m}\right) \cap R(\varnothing)=R(\varnothing)$ и утверждение очевидно. Следовательно, условие а) проверено.

Проверим условие b). Воспользуемся следуюшим утверждением.

Лемма 18 (Артина-Рисса [14]). Пусть $R$ - нётерово коммутативное кольцо с единицей, $M$ - левый унитарный нётеров $R$-модуль и а - идеал алгебры $R$. Тогда для любого $R$-подмодуля $P$ модуля $M$ существует число $k \in \mathbb{N}$ такое, что для любого $m \geqslant k$ выполнено $a^{m} M \cap P=a^{m-k}\left(a^{k} M \cap P\right)$.

СлЕДСТвИЕ 3. Пусть $P$ есть а-подмодуль модуля $M$ и $P_{i} \subset a^{i} M, Q_{i}=$ $P_{1}+\cdots+P_{i}, \quad i \in \mathbb{N} . \quad$ Тогда иепочка подмодулей $Q_{1} \subset \cdots \subset Q_{i} \subset \cdots$ стабилизируется.

ДокАЗАТЕЛЬСТво. В силу нётеровости $R$-модуля $M$ цепочка $R Q_{1} \subset \ldots$ $\cdots \subset R Q_{i} \subset \cdots$ стабилизируется. Пусть $s$ - такое число, что $R Q_{s}=R Q_{r}$ при $r \geqslant s$. По лемме Артина-Рисса существует такое число $k$, что $a^{k+1} M \cap$ $R Q_{s}=a\left(a^{k} M \cap R Q_{s}\right)$. Пусть $r$ - некоторое число, большее или равное $s$. Так как $Q_{r}$ есть $a$-модуль, то $a R Q_{r} \subset Q_{r}$. Имеем

$$
a^{k+1} M \cap Q_{r} \subset a^{k+1} M \cap R Q_{r}=a\left(a^{k} M \cap R Q_{r}\right) \subset a^{k+1} \cap Q_{r} .
$$


Отсюда получаем $a^{k+1} M \cap R Q_{r}=a^{k+1} M \cap Q_{r}$. Пусть $r_{1}, r_{2} \geqslant s_{0}=\max \{s, k\}$.

Предположим, что $f \in Q_{r_{1}}$. Тогда $f=f_{1}+f_{2}, f_{1} \in Q_{s_{0}}$ и $f_{2} \in P_{s_{0}+1}+\cdots$ $\cdots+P_{r_{1}} \subset a^{s_{0}+1} M \subset a^{k+1} M$. Имеем

$$
f_{2} \in a^{k+1} M \cap Q_{r_{1}}=a^{k+1} M \cap R Q_{r_{1}}=a^{k+1} M \cap R Q_{r_{2}}=a^{k+1} M \cap Q_{r_{2}} .
$$

Так как $r_{2} \geqslant s_{0}$, то $f_{1} \in Q_{s_{0}} \subset Q_{r_{2}}$ и $f=f_{1}+f_{2} \in Q_{r_{2}}$. Обратное включение доказывается аналогично. Следствие доказано.

Из этого следствия непосредственно получается

СЛЕДСТВИЕ 4. Пусть $P$ есть а-подмодуль модуля $M$ u $P_{i} \subset a^{m_{i}} M, Q_{i}=$ $P_{1}+\cdots+P_{i}, \quad i \in \mathbb{N}, \lim _{i \rightarrow \infty} m_{i}=\infty$. Тогда чепочка подмодулей $Q_{1} \subset \cdots$ $\cdots \subset Q_{i} \subset \cdots$ стабилизируется.

Пусть теперь $f_{1}, \ldots, f_{i}, \ldots$ - цепочка элементов из $R(\varnothing)$, бесконечно погружающаяся относительно фильтрации $(16), P_{i}$ есть $\left(T_{2}, \sigma_{0}\right)$-пространство, порожденное элементом $f_{i}$, и $\bar{P}_{i}$ есть $\left(T_{2}, \sigma_{0}\right)$-пространство, порожденное элементами $f_{1}, \ldots, f_{i}$.

Пространство $R\left(S_{n}\right)$ является нётеровым модулем над кольцом $\operatorname{Tr}(\varnothing)$. Действительно, применим теорему Ширшова о высоте к матричным алгебрам $\mathrm{GM}_{i}(\varnothing)$. Затем преобразуем произведения $u^{2 r_{i}}=\left(u^{2}\right)^{r_{i}}$ по теореме Гамильтона-Келли, примененной к матрице $u^{2}$ (у нее всегда четная $z$-степень), и получим требуемое утверждение. Кольцо $\operatorname{Tr}(\varnothing)$ само нётерово как конечно порожденная коммутативная алгебра над полем.

Пусть $a$ - идеал в алгебре $\operatorname{Tr}(\varnothing)$, порожденный произведениями $g_{1} \ldots g_{n}$, где $g_{i} \in \operatorname{Tr} \operatorname{GM}_{i}(\varnothing) \cap \operatorname{Tr}(\varnothing)$ - элемент вида (14) или (15). Тогда $a^{m} R\left(S_{n}\right)=I\left(C_{1}^{m}, \ldots\right.$ $\left.\ldots, C_{n}^{m}\right) \cap R\left(S_{n}\right)$.

Выберем некоторую допустимую пару $(\sigma, \eta)$ такую, что $|\sigma|=|\eta|=n$. Из свойства центральных полиномов Размыслова и леммы 14 следует, что $P_{i}^{(\sigma, \eta)}$ есть $a$-модуль. В силу п. 4) леммы 15 получаем $P_{i}^{(\sigma, \eta)} \subset a^{m_{i}} R\left(S_{n}\right)$, где $\lim _{i \rightarrow \infty} m_{i}=$ $\infty$. Пусть $Q_{i}^{(\sigma, \eta)}=P_{1}^{(\sigma, \eta)}+\cdots+P_{i}^{(\sigma, \eta)}$. Так как $P_{1}+\cdots+P_{i}=\bar{P}_{i}$, то в силу п. 2$)$ леммы 15 имеем

$$
Q_{i}^{(\sigma, \eta)}=P_{1}^{(\sigma, \eta)}+\cdots+P_{i}^{(\sigma, \eta)} \subset \bar{P}_{i}^{(\sigma, \eta)} .
$$

По следствию 4 цепочка $Q_{1}^{(\sigma, \eta)} \subset \cdots \subset Q_{i}^{(\sigma, \eta)} \subset \cdots$ стабилизируется, т. е. сушествует некоторое $s$ такое, что $Q_{r}^{(\sigma, \eta)}=Q_{s}^{(\sigma, \eta)}$ при $r \geqslant s$. Отсюда получаем

$$
P_{r}^{(\sigma, \eta)} \subset Q_{r}^{(\sigma, \eta)}=Q_{s}^{(\sigma, \eta)} \subset \bar{P}_{s}^{(\sigma, \eta)} .
$$

Так как количество допустимых пар $(\sigma, \eta)$ конечно, то число $s$ можно выбрать так, что включение $P_{r}^{(\sigma, \eta)} \subset \bar{P}_{s}^{(\sigma, \eta)}$ при $r \geqslant s$ выполнено для всех допустимых пар $(\sigma, \eta)$ таких, что $|\sigma|=|\eta|=n$. Тогда по п. 3$)$ леммы 15 получаем $P_{r} \subset \bar{P}_{s}$. Отсюда $f_{r} \in \bar{P}_{s}$. Пункт b) леммы проверен.

Следствие 2 и приведенные выше утверждения дают следующий основной результат.

ТеОрема 7. Любое T-пространство алгебры $K\langle X\rangle$, где $X-$ счетный алфавит и $K$ - поле нулевой характеристики, конечно базируемо. 


\section{Список литературы}

1. Кемер А.Р. О нематричных многообразиях // Алгебра и логика. 1980. Т. 19. №3. C. $255-283$.

2. Кемер А.P. Шпехтовость $T$-идеалов со степенным ростом коразмерностей // Сиб. матем. журн. 1978. Т. 19. № 1. С. 54-69.

3. Кемер А.Р. Многообразия и $Z_{2}$-градуированные алгебры // Изв. АН СССР. Сер. матем. 1984. Т. 48. № 5. С. 1042-1059.

4. Щиголев В. В. Примеры бесконечно базируемых T-пространств // Матем. сб. 2000. T. 191. № 3. C. $143-160$.

5. Щиголев B. В. Примеры бесконечно базируемых $T$-идеалов // Фунд. и прикл. математика. 1999. Т. 5. №1. С. 307-312.

6. Кемер А.Р. Конечная базируемость тождеств ассоциативных алгебр // Алгебра и логика. 1987. № 5. С. 597-641.

7. Кемер А.Р. Тождества конечно порожденных алгебр над бесконечным полем // Изв. АН СССР. Сер. матем. 1990. Т. 54. № 4. С. 726-753.

8. Гришин А.В. О конечной базируемости систем обобщенных многочленов // Изв. АН CССР. Сер. матем. 1990. Т. 54. № 5. С. 889-927.

9. Гришин $A . B$. О конечной базируемости абстрактных $T$-пространств // Фунд. и прикл. математика. 1995. Т. 1. №3. С. 669-700.

10. Гришин $A . B$. Примеры не конечной базируемости $T$-пространств и $T$-идеалов в характеристике 2 // Фунд. и прикл. математика. 1999. Т. 5. № 1. С. 101-118.

11. Белов А.Я. О нешпехтовых многообразиях // Фунд. и прикл. математика. 1999. Т. 5. № 1. C. $47-66$.

12. Джееймс Г. Теория представлений симметрической группы. М.: Мир, 1980.

13. Херстейн И. Некоммутативные кольца. М.: Мир, 1972.

14. Атья М., Макдональд И. Введение в коммутативную алгебру. М.: Мир, 1972.

15. Размыслов Ю. П. Введение в теорию алгебр и их представлений. М.: Изд-во МГУ, 1991.

16. Белов А.Я. Контрпримеры к проблеме Шпехта // Матем. сб. 2000. Т. 191. №3. C. $13-24$.

Московский государственный университет

Поступило в редакцию

им. М. В. Ломоносова

02.VI. 2000 